\title{
A direct discontinuous Galerkin method for fractional convection-diffusion and Schrödinger type equations
}

\author{
Tarek Aboelenen \\ Department of Mathematics, Assiut University, Assiut 71516, Egypt
}

\begin{abstract}
A direct discontinuous Galerkin (DDG) finite element method is developed for solving fractional convectiondiffusion and Schrödinger type equations with a fractional Laplacian operator of order $\alpha(1<\alpha<2)$. The fractional operator of order $\alpha$ is expressed as a composite of second order derivative and a fractional integral of order $2-\alpha$. These problems have been expressed as a system of parabolic equation and low order integral equation. This allows us to apply the DDG method which is based on the direct weak formulation for solutions of fractional convection-diffusion and Schrödinger type equations in each computational cell, letting cells communicate via the numerical flux $\left(\partial_{x} u\right)^{*}$ only. Moreover, we prove stability and optimal order of convergence $O\left(h^{N+1}\right)$ for the general fractional convection-diffusion and Schrödinger problems where $h, N$ are the space step size and polynomial degree. The DDG method has the advantage of easier formulation and implementation as well as the high order accuracy. Finally, numerical experiments confirm the theoretical results of the method.
\end{abstract}

Keywords: fractional convection-diffusion equations, fractional Schrödinger type equations, direct discontinuous Galerkin method, stability, error estimates.

\section{Introduction}

Fractional calculus is a useful tool in various areas of physics and engineering [1, 2, 3, 4, Several examples of applications can be found in wide areas, such as fractals 2, kinetic theories of systems with chaotic dynamics [5, 6, 7], pseudochaotic dynamics [8, anomalous transport [9, electrochemistry [10] and image processing [11, etc.

In this paper, we consider the fractional convection-diffusion equation

$$
\begin{aligned}
& \frac{\partial u}{\partial t}+\varepsilon(-\Delta)^{\frac{\alpha}{2}} u+\frac{\partial}{\partial x} f(u)=0, \quad x \in \mathbb{R}, t \in(0, T], \\
& u(x, 0)=u_{0}(x), \quad x \in \mathbb{R} .
\end{aligned}
$$

Email address: tarek.aboelenen@aun.edu.eg (Tarek Aboelenen) 
The generalized nonlinear fractional Schrödinger equation

$$
\begin{aligned}
& i \frac{\partial u}{\partial t}-\varepsilon_{1}(-\Delta)^{\frac{\alpha}{2}} u+\varepsilon_{2} f\left(|u|^{2}\right) u=0, \quad x \in \mathbb{R}, t \in(0, T], \\
& u(x, 0)=u_{0}(x), \quad x \in \mathbb{R},
\end{aligned}
$$

and the strongly coupled nonlinear fractional Schrödinger equations

$$
\begin{aligned}
& i \frac{\partial u}{\partial t}-\varepsilon_{1}(-\Delta)^{\frac{\alpha}{2}} u+\varpi_{1} u+\varpi_{2} v+\varepsilon_{2} f\left(|u|^{2},|v|^{2}\right) u=0, \quad x \in \mathbb{R}, t \in(0, T], \\
& i \frac{\partial v}{\partial t}-\varepsilon_{3}(-\Delta)^{\frac{\alpha}{2}} v+\varpi_{2} u+\varpi_{1} v+\varepsilon_{4} g\left(|u|^{2},|v|^{2}\right) v=0, \quad x \in \mathbb{R}, t \in(0, T], \\
& u(x, 0)=u_{0}(x), \quad x \in \mathbb{R}, \\
& v(x, 0)=v_{0}(x), \quad x \in \mathbb{R},
\end{aligned}
$$

and homogeneous boundary conditions. $f(u)$ and $g(u)$ are arbitrary (smooth) nonlinear real functions and $\varepsilon, \varepsilon_{i}$, $i=1,2,3,4$ are a real constants, $\varpi_{1}$ is normalized birefringence constant and $\varpi_{2}$ is the linear coupling parameter which accounts for the effects that arise from twisting and elliptic deformation of the fiber [12. The fractional Laplacian $-(-\Delta)^{\frac{\alpha}{2}}$, which can be defined using Fourier analysis as [13, 14, 15]

$$
-(-\Delta)^{\frac{\alpha}{2}} u(x, t)=\mathcal{F}^{-1}\left(|\xi|^{\alpha} \hat{u}(\xi, t)\right)
$$

where $\mathcal{F}$ is the Fourier transform.

The equation (1.1) is involved in many different physical problems, such as such as geomorphology [16, 17, overdriven detonations in gases [17, 18, signal processing [19], and anomalous diffusion in semiconductor growth [20. Numerical studies of fractional convection diffusion equations have attracted a lot of interest in recent years. Several authors have proposed a variety of high-order finite difference schemes for solving time-fractional convection-diffusion equations, for examples [21, 22, 23, 24, and solving space-fractional convection-diffusion equations [25, 26]. Furthermore, numerical methods for fractional diffusion problems and financial models with fractional Laplacian operators or Riesz fractional derivatives have been studied in a number of papers, such as [27, 28, 15, 29, 30].

The equations 1.2 and 1.3 arise in many physical fields, especially in in fluid mechanics, nonlinear optics, solid state physics and plasma waves and for two interacting nonlinear packets in a dispersive and conservative system, see, e.g., 31, 32, 33, 34 and reference therein. In recent years, developing various numerical algorithms for solving these problems has received much attention. Wang and Huang [35] studied an energy conservative CrankNicolson difference scheme for nonlinear Riesz space-fractional Schrödinger equation. Yang [36] proposed a class of linearized energy-conserved finite difference schemes for nonlinear space-fractional Schrödinger equation. Galerkin finite element method for nonlinear fractional Schrödinger equations were considered 37. Ran and Zhang 34 proposed a conservative difference scheme for solving the strongly coupled nonlinear fractional Schrödinger equations. A numerical study based on an implicit fully discrete LDG for the time-fractional coupled Schrödinger 
systems is presented [38].

The discontinuous Galerkin (DG) method is a class of finite element methods using discontinuous, piecewise polynomials as the solution and the test spaces in the spatial direction. There have been various DG methods suggested in the literature to solve diffusion problem, including the method originally proposed by Bassi and Rebay [39] for compressible Navier-Stokes equations, its generalization called the local discontinuous Galerkin (LDG) methods introduced in [40] by Cockburn and Shu and further studied in [41, 42]. For application of the method to fractional problems, Mustapha and McLean [43, 44, 45, 46, have developed and analyzed discontinuous Galerkin methods for time fractional diffusion and wave equations. Xu and Hesthaven [47] proposed a LDG method for fractional convection-diffusion equations. They proved stability and optimal order of convergence $N+1$ for the fractional diffusion problem when polynomials of degree $N$, and an order of convergence of $N+\frac{1}{2}$ is established for the general fractional convection-diffusion problem with general monotone flux for the nonlinear term. Aboelenen and El-Hawary [48] proposed a high-order nodal discontinuous Galerkin method for a linearized fractional Cahn-Hilliard equation. They proved stability and optimal order of convergence $N+1$ for the linearized fractional Cahn-Hilliard problem. A nodal discontinuous Galerkin method was developed to solve the nonlinear Riesz space fractional Schrödinger equation and the strongly coupled nonlinear Riesz space fractional Schrödinger equations [49]. They proved, for both problems, $L^{2}$ stability and optimal order of convergence $O\left(h^{N+1}\right)$. Huang et al. [50] solved and analyzed the time fractional diffusion equations by using a fully discrete DDG method.

The key of the local discontinuous Galerkin method for the fractional convection-diffusion and Schrödinger type equations [47, 49] is to rewrite the fractional operator as a composite of first order derivatives and a fractional integral and convert these problems into a system of low order equations by introducing an auxiliary variable. By solving the system, one obtains the solutions of the fractional convection-diffusion and Schrödinger type equations. The shortcoming is computational cost larger whether you use explicit and implicit step to solve the fully discrete system by the LDG method. Other DG method is called the DDG method introduced in [51, 52, 53. which is based on weak formulation for the solution of the parabolic equation in each computational cell and cells communicate via the numerical flux $\left(\partial_{x} u\right)^{*}$ only. Here, we rewrite the fractional Laplacian operator as a composite of second order derivative and a fractional integral and convert the fractional convection-diffusion and Schrödinger type equations into a system of parabolic equation and low order integral equation. This allows us to apply the DDG method which is based on the direct weak formulation of the equations (1.1)-(1.3) and the construct of the suitable numerical flux on the cell edges. This method is called DDG method for not introducing any auxiliary variables in contrast to the LDG method [47, 49].

The rest of this paper is organized as follows. Section 2 introduce some basic definitions and recall a few central results. In section 3 , we derive the DDG formulation for the fractional convection-diffusion problem. We present a stability and convergence analysis for the fractional convection-diffusion equations in section 4 . We derive the DDG formulation for the nonlinear fractional Schrödinger equation in section 5. Then we prove a theoretical result of $L^{2}$ stability for the nonlinear case as well as an error estimate for the linear case in section 6 . In 
section 7 we present a DDG method for the strongly coupled nonlinear fractional Schrödinger equations and give a theoretical result of $L^{2}$ stability for the nonlinear case and an error estimate for the linear case. Section 8 presents some numerical examples to illustrate the efficiency of the scheme. A few concluding remarks are offered in section 9 ,

\section{Preliminary definitions}

In this section, we make some preparation including the definitions of fractional derivatives [54] and associated functional setting for the subsequent numerical schemes and theoretical analysis.

Apart from the definitions of the fractional Laplacian based on the Fourier and the integral form, it can also be defined using ideas of fractional calculus [13, 14, 15], as

$$
\frac{\partial^{\alpha}}{\partial|x|^{\alpha}} u(x, t)=-(-\Delta)^{\frac{\alpha}{2}} u(x, t)=-\frac{{ }_{-\infty}^{C} \mathcal{D}_{x}^{\alpha} u(x, t)+{ }_{x}^{C} \mathcal{D}_{\infty}^{\alpha} u(x, t)}{2 \cos \left(\frac{\pi \alpha}{2}\right)},
$$

where ${ }_{-\infty}^{C} \mathcal{D}_{x}^{\alpha}$ and ${ }_{x}^{C} \mathcal{D}_{\infty}^{\alpha}$ refer to the left and right Caputo fractional derivatives, respectively, of $\alpha$ th order. This definition is also known as a Riesz derivative. To prepare we introduce a few definitions and recall some properties of fractional integrals and derivatives.

The left-sided and right-sided Riemann-Liouville integrals of order $\alpha$, when $0<\alpha<1$, are defined, respectively, as

$$
\left({ }_{-\infty}^{R L} \mathcal{I}_{x}^{\alpha} f\right)(x)=\frac{1}{\Gamma(\alpha)} \int_{-\infty}^{x} \frac{f(s) d s}{(x-s)^{1-\alpha}}, \quad x>-\infty
$$

and

$$
\left({ }_{x}^{R L} \mathcal{I}_{\infty}^{\alpha} f\right)(x)=\frac{1}{\Gamma(\alpha)} \int_{x}^{\infty} \frac{f(s) d s}{(s-x)^{1-\alpha}}, \quad x<\infty
$$

where $\Gamma$ represents the Euler Gamma function. The corresponding inverse operators, i.e., the left-sided and right-sided fractional derivatives of order $\alpha$, are then defined based on $(2.2)$ and $(2.3)$, as

$$
\left({ }_{-\infty}^{R L} \mathcal{D}_{x}^{\alpha} f\right)(x)=\frac{d}{d x}\left({ }_{-\infty}^{R L} \mathcal{I}_{x}^{1-\alpha} f\right)(x)=\frac{1}{\Gamma(1-\alpha)} \frac{d}{d x} \int_{-\infty}^{x} \frac{f(s) d s}{(x-s)^{\alpha}}, \quad x>-\infty,
$$

and

$$
\left({ }_{x}^{R L} \mathcal{D}_{\infty}^{\alpha} f\right)(x)=\frac{-d}{d x}\left({ }_{x}^{R L} \mathcal{I}_{\infty}^{1-\alpha} f\right)(x)=\frac{1}{\Gamma(1-\alpha)}\left(\frac{-d}{d x}\right) \int_{x}^{\infty} \frac{f(s) d s}{(s-x)^{\alpha}}, \quad x<\infty .
$$

This allows for the definition of the left and right Riemann-Liouville fractional derivatives of order $\alpha(n-1<$ $\alpha<n), n \in \mathbb{N}$ as

$$
\left({ }_{-\infty}^{R L} \mathcal{D}_{x}^{\alpha} f\right)(x)=\left(\frac{d}{d x}\right)^{n}\left({ }_{-\infty}^{R L} \mathcal{I}_{x}^{n-\alpha} f\right)(x)=\frac{1}{\Gamma(n-\alpha)}\left(\frac{d}{d x}\right)^{n} \int_{-\infty}^{x} \frac{f(s) d s}{(x-s)^{-n+1+\alpha}}, \quad x>-\infty,
$$


and

$$
\left({ }_{x}^{R L} \mathcal{D}_{\infty}^{\alpha} f\right)(x)=\left(\frac{-d}{d x}\right)^{n}\left({ }_{x}^{R L} \mathcal{I}_{\infty}^{n-\alpha} f\right)(x)=\frac{1}{\Gamma(n-\alpha)}\left(\frac{-d}{d x}\right)^{n} \int_{x}^{\infty} \frac{f(s) d s}{(s-x)^{-n+1+\alpha}}, \quad x<\infty .
$$

Furthermore, the corresponding left-sided and right-sided Caputo derivatives of order $\alpha(n-1<\alpha<n)$ are obtained as

$$
\left({ }_{-\infty}^{C} \mathcal{D}_{x}^{\alpha} f\right)(x)=\left({ }_{-\infty}^{R L} \mathcal{I}_{x}^{n-\alpha} \frac{d^{n} f}{d x^{n}}\right)(x)=\frac{1}{\Gamma(n-\alpha)} \int_{-\infty}^{x} \frac{f^{(n)}(s) d s}{(x-s)^{-n+1+\alpha}}, \quad x>-\infty,
$$

and

$$
\left({ }_{x}^{C} \mathcal{D}_{\infty}^{\alpha} f\right)(x)=(-1)^{n}\left({ }_{x}^{R L} \mathcal{I}_{\infty}^{n-\alpha} \frac{d^{n} f}{d x^{n}}\right)(x)=\frac{1}{\Gamma(n-\alpha)} \int_{x}^{\infty} \frac{(-1)^{n} f^{(n)}(s) d s}{(s-x)^{-n+1+\alpha}}, \quad x<\infty .
$$

If $\alpha<0$, the fractional Laplacian becomes the fractional integral operator. In this case, for any $0<\mu<1$, we define

$$
\Delta_{-\mu / 2} u(x)=-\frac{{ }_{\infty}^{C} \mathcal{D}_{x}^{-\mu} u(x)+{ }_{x}^{C} \mathcal{D}_{\infty}^{-\mu} u(x)}{2 \cos \left(\frac{\pi(2-\mu)}{2}\right)}=\frac{{ }_{\infty}^{C} \mathcal{D}_{x}^{-\mu} u(x)+{ }_{x}^{C} \mathcal{D}_{\infty}^{-\mu} u(x)}{2 \cos \left(\frac{\pi \mu}{2}\right)}=\frac{{ }_{-\infty}^{R L} \mathcal{I}_{x}^{-\mu} u(x)+{ }_{x}^{R L} \mathcal{I}_{\infty}^{-\mu} u(x)}{2 \cos \left(\frac{\pi \mu}{2}\right)} .
$$

When $1<\alpha<2$, using (2.8), 2.9$)$ and $(2.10)$, we can rewrite the fractional Laplacian in the following form:

$$
-(-\Delta)^{\frac{\alpha}{2}} u(x)=\Delta_{\frac{(\alpha-2)}{2}}\left(\frac{d^{2} u(x)}{d x^{2}}\right) .
$$

To carry out the analysis, we introduce the appropriate fractional spaces.

Definition 2.1. (The right and left fractional spaces [55]). We define the seminorm

$$
\begin{aligned}
& |u|_{J_{R}^{\alpha}(\mathbb{R})}=\left\|{ }_{x}^{R L} \mathcal{D}_{x_{R}}^{\alpha} u\right\|_{L^{2}(\mathbb{R})}, \\
& |u|_{J_{L}^{\alpha}(\mathbb{R})}=\left\|{ }_{x_{L}}^{R L} \mathcal{D}_{x}^{\alpha} u\right\|_{L^{2}(\mathbb{R})} .
\end{aligned}
$$

and the norm

$$
\begin{aligned}
& \|u\|_{J_{R}^{\alpha}(\mathbb{R})}=\left(|u|_{J_{R}^{\alpha}(\mathbb{R})}^{2}+\|u\|_{L^{2}(\mathbb{R})}^{2}\right)^{\frac{1}{2}}, \\
& \|u\|_{J_{L}^{\alpha}(\mathbb{R})}=\left(|u|_{J_{L}^{\alpha}(\mathbb{R})}^{2}+\|u\|_{L^{2}(\mathbb{R})}^{2}\right)^{\frac{1}{2}},
\end{aligned}
$$

and let the two spaces $J_{R}^{\alpha}(\mathbb{R})$ and $J_{L}^{\alpha}(\mathbb{R})$ denote the closure of $C_{0}^{\infty}(\mathbb{R})$ with respect to $\|\cdot\|_{J_{R}^{\alpha}(\mathbb{R})}$ and $\|\cdot\|_{J_{L}^{\alpha}(\mathbb{R})}$ respectively. 
Definition 2.2. (symmetric fractional space [55]). We define the seminorm

$$
\|u\|_{J_{S}^{\alpha}(\mathbb{R})}=\left|\left({ }_{x_{L}}^{R L} \mathcal{D}_{x}^{\alpha} u,{ }_{x}^{R L} \mathcal{D}_{x_{R}}^{\alpha} u\right)_{L^{2}(\mathbb{R})}\right|^{\frac{1}{2}},
$$

and the norm

$$
\|u\|_{J_{S}^{\alpha}(\mathbb{R})}=\left(|u|_{J_{S}^{\mu}(\mathbb{R})}^{2}+\|u\|_{L^{2}(\mathbb{R})}^{2}\right)^{\frac{1}{2}} .
$$

and let $J_{S}^{\alpha}(\mathbb{R})$ denote the closure of $C_{0}^{\infty}(\mathbb{R})$ with respect to $\|\cdot\|_{J_{S}^{\alpha}(\mathbb{R})}$.

Lemma 2.1. (see [55]). For any $0<s<1$, the fractional integral satisfies the following property:

$$
\left({ }_{-\infty}^{R L} \mathcal{I}_{x}^{s} u,{ }_{x}^{R L} \mathcal{I}_{\infty}^{s} u\right)_{\mathbb{R}}=\cos (s \pi)|u|_{J_{L}^{-s}(\mathbb{R})}^{2}=\cos (s \pi)|u|_{J_{R}^{-s}(\mathbb{R})}^{2} .
$$

Lemma 2.2. For any $0<\mu<1$, the fractional integral satisfies the following property:

$$
\left(\Delta_{-\mu} u, u\right)_{\mathbb{R}}=|u|_{J_{L}^{-\mu}(\mathbb{R})}^{2}=|u|_{J_{R}^{-\mu}(\mathbb{R})}^{2} .
$$

Generally, we consider the problem in a bounded domain instead of $\mathbb{R}$. Hence, we restrict the definition to the domain $\Omega=[a, b]$.

Definition 2.3. Define the spaces $J_{R, 0}^{\alpha}(\Omega), J_{L, 0}^{\alpha}(\Omega), J_{S, 0}^{\alpha}(\Omega)$ as the closures of $C_{0}^{\infty}(\Omega)$ under their respective norms.

Lemma 2.3. (fractional Poincaré-Friedrichs, [55]). For $u \in J_{L, 0}^{\alpha}(\Omega)$ and $\alpha \in \mathbb{R}$, we have

$$
\|u\|_{L^{2}(\Omega)} \leq C|u|_{J_{L, 0}^{\alpha}(\Omega)},
$$

and for $u \in J_{R, 0}^{\alpha}(\Omega)$, we have

$$
\|u\|_{L^{2}(\Omega)} \leq C|u|_{J_{R, 0}^{\alpha}(\Omega)}
$$

Lemma 2.4. (See [56]) For any $0<\mu<1$, the fractional integration operator ${ }_{a}^{R L} \mathcal{I}_{x}^{\mu}$ is bounded in $L^{2}(\Omega)$ :

$$
\left\|_{a}^{R L} \mathcal{I}_{x}^{\mu} u\right\|_{L^{2}(\Omega)} \leq K\|u\|_{L^{2}(\Omega)}
$$

The fractional integration operator ${ }_{x}^{R L} \mathcal{I}_{b}^{\mu}$ is bounded in $L^{2}(\Omega)$ :

$$
\left\|{ }_{x}^{R L} \mathcal{I}_{b}^{\mu} u\right\|_{L^{2}(\Omega)} \leq K\|u\|_{L^{2}(\Omega)}
$$

Lemma 2.5. (See [49]) The fractional integration operator $\Delta_{-\mu}$ is bounded in $L^{2}(\Omega)$ :

$$
\left\|\Delta_{-\mu} u\right\|_{L^{2}(\Omega)} \leq K\|u\|_{L^{2}(\Omega)} .
$$




\section{The DDG scheme for the fractional convection-diffusion equation}

In this section, we construct DDG method for the fractional convection-diffusion problem (1.1). So, we rewrite the Riesz fractional derivative of order $\alpha(1<\alpha<2)$ as a composite of second order derivative and low order fractional integral operator. Since the integral operator naturally connects the discontinuous function, we need not add a penalty term or introduce a numerical fluxes for the integral equation.

We introduce the auxiliary variables $p, q$ and set

$$
p=\Delta_{(\alpha-2) / 2} q, \quad q=\frac{\partial^{2}}{\partial x^{2}} u,
$$

then, the nonlinear fractional convection-diffusion problem can be rewritten as

$$
\begin{aligned}
& \frac{\partial u}{\partial t}-\varepsilon p+\frac{\partial}{\partial x} f(u)=0, \\
& p=\Delta_{(\alpha-2) / 2} q, \quad q=\frac{\partial^{2}}{\partial x^{2}} u .
\end{aligned}
$$

The weak solution formulation for this problem is to find a functions $u, p, q \in C\left(0, T ; H^{1}(\Omega)\right)$ such that for all $v, \psi, \phi \in H_{0}^{1}(\Omega)$

$$
\begin{aligned}
& \left(\frac{\partial u}{\partial t}, v\right)-\varepsilon(p, v)-\left(f(u), \partial_{x} v\right)=0, \\
& (p, \psi)=\left(\Delta_{(\alpha-2) / 2} q, \psi\right), \\
& (q, \phi)=-\left(\partial_{x} u, \partial_{x} \phi\right) .
\end{aligned}
$$

To discretize this weak formulation, we set up a partition of the domain $\Omega$ into $K$ non-overlapping elements such that $\Omega=\bigcup_{k=1}^{K} D^{k}$ with mesh $D^{k}=\left[x_{k-\frac{1}{2}}, x_{k+\frac{1}{2}}\right], \Delta x_{k}=x_{k+\frac{1}{2}}-x_{k-\frac{1}{2}}$ and $k=1, \ldots, K$. Let $u_{h}, p_{h}, q_{h} \in V_{k}^{N}$ be the approximation of $u, p, q$ respectively, where the approximation space is defined as

$$
V_{k}^{N}=\left\{v: v_{k} \in \mathbb{P}^{N}\left(D^{k}\right), \forall D^{k} \in \Omega\right\}
$$

where $\mathbb{P}^{N}\left(D^{k}\right)$ denotes the set of polynomials of degree up to $N$ defined on the element $D^{k}$.

We define the local inner product and $L^{2}\left(D^{k}\right)$ norm

$$
\begin{aligned}
& (u, v)_{D^{k}}=\int_{D^{k}} u v d x, \quad\|u\|_{D^{k}}^{2}=(u, u)_{D^{k}}, \\
& (u, v)=\sum_{k=1}^{K}(u, v)_{D^{k}}, \quad\|u\|_{L^{2}(\Omega)}^{2}=\sum_{k=1}^{K}(u, u)_{D^{k}} .
\end{aligned}
$$

We define DDG scheme as follows: find $u_{h}, p_{h}, q_{h} \in V_{k}^{N}$, such that for all test functions $v, \psi, \phi \in V_{k}^{N}$,

$$
\begin{aligned}
& \left(\frac{\partial u_{h}}{\partial t}, v\right)_{D^{k}}-\varepsilon\left(p_{h}, v\right)_{D^{k}}+\left(\frac{\partial}{\partial x} f\left(u_{h}\right), v\right)_{D^{k}}=0, \\
& \left(p_{h}, \psi\right)_{D^{k}}=\left(\Delta_{(\alpha-2) / 2} q_{h}, \psi\right)_{D^{k}} \\
& \left(q_{h}, \phi\right)_{D^{k}}=\left(\frac{\partial^{2}}{\partial x^{2}} u_{h}, \phi\right)_{D^{k}} .
\end{aligned}
$$


To complete the DDG scheme, we introduce some notation and the numerical flux.

Define

$$
\{u\}=\frac{u^{+}+u^{-}}{2}, \quad[u]=u^{+}-u^{-} .
$$

The numerical flux involves the average $\left\{\partial_{x} u\right\}$ and the jumps of even order derivatives of $u[52$ :

$$
\left(\partial_{x} u\right)^{*}=\frac{\beta_{0}}{h}[u]+\left\{\partial_{x} u\right\}+\beta_{1} h\left[\partial_{x}^{2} u\right] .
$$

where $\beta_{0}$ and $\beta_{1}$ are chosen to ensure the stability and accuracy of the scheme.

The idea of the DDG method is to enforce the weak formulation (3.3) in such a way that both $u_{h}$ and $\phi$ are approximated in $V_{k}^{N}$. The discontinuous nature of numerical solutions and test functions crossing interfaces necessarily requires some interface corrections, leading to the following:

$$
\begin{aligned}
& \left(\left(u_{h}\right)_{t}, v\right)_{D^{k}}-\varepsilon\left(p_{h}, v\right)_{D^{k}}-\left(f\left(u_{h}\right), \partial_{x} v\right)_{D^{k}}+\left(\hat{n} . f\left(u_{h}\right)^{*}, v\right)_{\partial D^{k}}=0, \\
& \left(p_{h}, \psi\right)_{D^{k}}=\left(\Delta_{(\alpha-2) / 2} q_{h}, \psi\right)_{D^{k}}, \\
& \left(q_{h}, \phi\right)_{D^{k}}=-\left(\partial_{x} u_{h}, \partial_{x} \phi\right)_{D^{k}}-\left(\left(\partial_{x} u_{h}\right)^{*}[\phi]+\left\{\partial_{x} \phi\right\}\left[u_{h}\right]\right)_{k+\frac{1}{2}} .
\end{aligned}
$$

\section{Stability and error estimates}

In the following we discuss stability and accuracy of the proposed scheme, for the fractional diffusion problem and the nonlinear fractional convection-diffusion problem.

\subsection{Stability analysis}

In order to carry out the analysis of the DDG scheme, we have the following results.

Definition 4.1. (Admissibility [52]). We call a numerical flux $\left(\partial_{x} u_{h}\right)^{*}$ of the form (3.9) admissible if there exists a $\gamma \in(0,1)$ and $0<\mu \leq 1$ such that

$$
\gamma\left(\partial_{x} u_{h}, \partial_{x} u_{h}\right)+\sum_{k=1}^{K}\left(\left(\partial_{x} u_{h}\right)^{*}\left[u_{h}\right]+\left\{\partial_{x} u_{h}\right\}\left[u_{h}\right]\right)_{k+\frac{1}{2}} \geq \mu \sum_{k=1}^{K} \frac{\left[u_{h}\right]_{k+\frac{1}{2}}^{2}}{h} .
$$

holds for any piecewise polynomials of degree $N$, i.e., $u \in V_{k}^{N}$

Theorem 4.1. ( $L^{2}$ stability). The semidiscrete scheme 3.10$)$ is $L^{2}$ stable, and for all $T>0$ its solution satisfies

$$
\left\|u_{h}(x, T)\right\|_{L^{2}(\Omega)} \leq c\left\|u_{0}(x)\right\|_{L^{2}(\Omega)} .
$$

Proof. Set $(v, \psi, \phi)=\left(u_{h}, p_{h}-q_{h}, u_{h}\right)$ in 3.10 , we get

$$
\begin{aligned}
& \left(\left(u_{h}\right)_{t}, u_{h}\right)_{D^{k}}-\left(f\left(u_{h}\right), \partial_{x} u_{h}\right)_{D^{k}}+\left(\hat{n} \cdot f\left(u_{h}\right)^{*}, u_{h}\right)_{\partial D^{k}}+\left(p_{h}, p_{h}\right)_{D^{k}}+\left(\Delta_{(\alpha-2) / 2} q_{h}, q_{h}\right)_{D^{k}} \\
& =\left(p_{h}, q_{h}\right)_{D^{k}}+\varepsilon\left(p_{h}, u_{h}\right)_{D^{k}}-\left(q_{h}, u_{h}\right)_{D^{k}}-\left(\partial_{x} u_{h}, \partial_{x} u_{h}\right)_{D^{k}}+\left(\Delta_{(\alpha-2) / 2} q_{h}, p_{h}\right)_{D^{k}}-\left(\left(\partial_{x} u_{h}\right)^{*}\left[u_{h}\right]+\left\{\partial_{x} u_{h}\right\}\left[u_{h}\right]\right)_{k+\frac{1}{2}}
\end{aligned}
$$


Define $\theta(u)=\int^{u} f(s) d s ;$ then

$$
\begin{aligned}
& \left(\left(u_{h}\right)_{t}, u_{h}\right)_{D^{k}}+\left(\hat{n} \cdot\left(f\left(u_{h}\right)^{*}, u_{h}\right)_{\partial D^{k}}-\left(\theta\left(u_{h}\right)\right)_{k+\frac{1}{2}}^{-}+\left(\theta\left(u_{h}\right)\right)_{k-\frac{1}{2}}^{+}+\left(p_{h}, p_{h}\right)_{D^{k}}+\left(\Delta_{(\alpha-2) / 2} q_{h}, q_{h}\right)_{D^{k}}\right. \\
& =\left(p_{h}, q_{h}\right)_{D^{k}}+\varepsilon\left(p_{h}, u_{h}\right)_{D^{k}}-\left(q_{h}, u_{h}\right)_{D^{k}}-\left(\partial_{x} u_{h}, \partial_{x} u_{h}\right)_{D^{k}}+\left(\Delta_{(\alpha-2) / 2} q_{h}, p_{h}\right)_{D^{k}}-\left(\left(\partial_{x} u_{h}\right)^{*}\left[u_{h}\right]+\left\{\partial_{x} u_{h}\right\}\left[u_{h}\right]\right)_{k+\frac{1}{2}} .
\end{aligned}
$$

Employing Young's inequality and Lemma 2.5, we obtain

$$
\begin{aligned}
& \left(\left(u_{h}\right)_{t}, u_{h}\right)_{D^{k}}+\left(\hat{n} \cdot\left(f\left(u_{h}\right)^{*}, u_{h}\right)_{\partial D^{k}}-\left(\theta\left(u_{h}\right)\right)_{k+\frac{1}{2}}^{-}+\left(\theta\left(u_{h}\right)\right)_{k-\frac{1}{2}}^{+}+\left(p_{h}, p_{h}\right)_{D^{k}}+\left(\Delta_{(\alpha-2) / 2} q_{h}, q_{h}\right)_{D^{k}}\right. \\
& \leq c_{3}\left\|u_{h}\right\|_{L^{2}\left(D^{k}\right)}^{2}+c_{1}\left\|p_{h}\right\|_{L^{2}\left(D^{k}\right)}^{2}+c_{2}\left\|q_{h}\right\|_{L^{2}\left(D^{k}\right)}^{2}-\left(\partial_{x} u_{h}, \partial_{x} u_{h}\right)_{D^{k}}-\left(\left(\partial_{x} u_{h}\right)^{*}\left[u_{h}\right]+\left\{\partial_{x} u_{h}\right\}\left[u_{h}\right]\right)_{k+\frac{1}{2}} .
\end{aligned}
$$

Recalling Lemma 2.3 , provided $c_{1}$ is sufficiently small such that $c_{1} \leq 1$, we obtain that

$$
\begin{aligned}
& \left(\left(u_{h}\right)_{t}, u_{h}\right)_{D^{k}}+\left(\hat{n} \cdot\left(f\left(u_{h}\right)^{*}, u_{h}\right)_{\partial D^{k}}-\left(\theta\left(u_{h}\right)\right)_{k+\frac{1}{2}}^{-}+\left(\theta\left(u_{h}\right)\right)_{k-\frac{1}{2}}^{+}\right. \\
& \leq c_{3}\left\|u_{h}\right\|_{L^{2}\left(D^{k}\right)}^{2}-\left(\partial_{x} u_{h}, \partial_{x} u_{h}\right)_{D^{k}}-\left(\left(\partial_{x} u_{h}\right)^{*}\left[u_{h}\right]+\left\{\partial_{x} u_{h}\right\}\left[u_{h}\right]\right)_{k+\frac{1}{2}},
\end{aligned}
$$

such that $\left(\hat{n} \cdot f\left(u_{h}\right)^{*}, u_{h}\right)_{\partial D^{k}}-\left(\theta\left(u_{h}\right)\right)_{k+\frac{1}{2}}^{-}+\left(\theta\left(u_{h}\right)\right)_{k-\frac{1}{2}}^{+} \geq 0$, summering over all elements, we immediately recover

$$
\left(\left(u_{h}\right)_{t}, u_{h}\right) \leq c_{3}\left\|u_{h}\right\|_{L^{2}(\Omega)}^{2}-\left(\partial_{x} u_{h}, \partial_{x} u_{h}\right)-\sum_{k=1}^{K}\left(\left(\partial_{x} u_{h}\right)^{*}\left[u_{h}\right]+\left\{\partial_{x} u_{h}\right\}\left[u_{h}\right]\right)_{k+\frac{1}{2}} .
$$

From the admissible condition 4.1 of the numerical flux defined in 3.9$)$, we obtain that

$$
\left(\left(u_{h}\right)_{t}, u_{h}\right)+(1-\gamma)\left\|\partial_{x} u_{h}\right\|_{L^{2}(\Omega)}^{2}+\mu \sum_{k=1}^{K} \frac{\left[u_{h}\right]_{k+\frac{1}{2}}^{2}}{h} \leq c_{3}\left\|u_{h}\right\|_{L^{2}(\Omega)}^{2} .
$$

Employing Gronwall's lemma, we obtain $\left\|u_{h}(x, T)\right\|_{L^{2}(\Omega)} \leq c\left\|u_{0}(x)\right\|_{L^{2}(\Omega)}$.

\subsection{Error estimates}

we list some inverse properties and special projections $\mathcal{P}^{+}$of the finite element space $V_{k}^{N}$ that will be used in our error analysis.

$$
\begin{aligned}
& \left(\mathcal{P}^{+} u-u, v\right)_{D^{k}}=0, \quad \forall v \in \mathbb{P}_{N}^{k-2}\left(D^{k}\right), \quad k=1, \ldots, K, \\
& \left(\partial_{x} \mathcal{P}^{-} u\right)^{*}=\left(\beta_{0} h^{-1}\left[\mathcal{P}^{-} u\right]+\left\{\partial_{x} \mathcal{P}^{-} u\right\}+\beta_{1} h\left[\partial_{x}^{2} \mathcal{P}^{-} u\right]\right)_{x_{k+\frac{1}{2}}}=\left(\partial_{x} u\left(x_{k+\frac{1}{2}}\right)\right)^{*} .
\end{aligned}
$$

Lemma 4.1. (See [53]) For $\left(\beta_{0}, \beta_{1}\right)$ such that $\beta_{0}>\Gamma\left(\beta_{1}\right)$, the projection $\mathcal{P}^{+}$defined in 4.8 exists, and

$$
\left\|\mathcal{P}^{+} u(.)-u(.)\right\|_{L^{2}(\Omega)} \leq C h^{N+1} .
$$


Let $\pi^{e}$ denote the projection error, then the following inequality [53]:

$$
\left\|\pi^{e}\right\|_{L^{2}(\Omega)}+h\left\|\pi^{e}\right\|_{\infty}+h^{\frac{1}{2}}\|\pi\|_{\Gamma_{h}} \leq C h^{N+1} .
$$

For any function $u_{h} \in V_{k}^{N}$, the following inverse inequalities hold [57]:

$$
\begin{aligned}
& \left\|\partial_{x} u_{h}\right\|_{L^{2}(\Omega)} \leq C h^{-1}\left\|u_{h}\right\|_{L^{2}\left(\Omega_{h}\right)}, \\
& \left\|u_{h}\right\|_{\Gamma_{h}} \leq C h^{-1 / 2}\left\|u_{h}\right\|_{L^{2}(\Omega)}, \\
& \left\|u_{h}\right\|_{\infty} \leq C h^{-1 / 2}\left\|u_{h}\right\|_{L^{2}(\Omega)},
\end{aligned}
$$

where $\Gamma_{h}$ denotes the set of interface points of all the elements, $D^{k}, k=1,2, \ldots, K$ and here and below $C$ is a positive constant (which may have a different value in each occurrence) depending solely on $\mathrm{u}$ and its derivatives but not of $h$.

Theorem 4.2. (Diffusion without convection $f(u)=0$ ). Let $u$ be the exact solutions of the problem (1.1), and let $u_{h}$ be the numerical solutions of the semi-discrete DDG scheme (3.10). Then for small enough $h$, we have the following error estimates:

$$
\left\|u-u_{h}\right\|_{L^{2}(\Omega)} \leq C h^{N+1},
$$

where the constant $C$ is dependent upon $T$ and some norms of the solutions.

Proof. We consider the fractional diffusion equation

$$
\frac{\partial u}{\partial t}+\varepsilon(-\Delta)^{\frac{\alpha}{2}} u=0 .
$$

It is easy to verify that the exact solution of the above 4.13 satisfies

$$
\left(u_{t}, v\right)_{D^{k}}-\varepsilon(p, v)_{D^{k}}+(p, \psi)_{D^{k}}-\left(\Delta_{(\alpha-2) / 2} q, \psi\right)_{D^{k}}+(q, \phi)_{D^{k}}+\left(\partial_{x} u, \partial_{x} \phi\right)_{D^{k}}+\left(\left(\partial_{x} u_{h}\right)^{*}[\phi]+\left\{\partial_{x} \phi\right\}\left[u_{h}\right]\right)_{k+\frac{1}{2}}=0 .
$$

Subtracting (4.14) from the fractional diffusion equation (3.10), we have the following error equation

$$
\begin{aligned}
& \left(\left(u-u_{h}\right)_{t}, v\right)_{D^{k}}-\varepsilon\left(p-p_{h}, v\right)_{D^{k}}+\left(p-p_{h}, \psi\right)_{D^{k}}-\left(\Delta_{(\alpha-2) / 2}\left(q-q_{h}\right), \psi\right)_{D^{k}} \\
& \quad+\left(q-q_{h}, \phi\right)_{D^{k}}+\left(\partial_{x}\left(u-u_{h}\right), \partial_{x} \phi\right)_{D^{k}}-\left(\left(\partial_{x}\left(u-u_{h}\right)\right)^{*}[\phi]+\left(\partial_{x} \phi\right)^{*}\left[u-u_{h}\right]\right)_{k+\frac{1}{2}}=0 .
\end{aligned}
$$

Denoting

$$
\begin{aligned}
& \pi=\mathcal{P}^{+} u-u_{h}, \quad \pi^{e}=\mathcal{P}^{+} u-u, \quad \epsilon=\mathcal{P}^{+} p-p_{h}, \quad \epsilon^{e}=\mathcal{P}^{+} p-p, \\
& \varphi^{e}=\mathcal{P}^{+} q-q, \quad \varphi=\mathcal{P}^{+} q-q_{h} .
\end{aligned}
$$

From the Galerkin orthogonality [4.15], we get

$$
\begin{aligned}
& \left(\left(\pi-\pi^{e}\right)_{t}, v\right)_{D^{k}}-\varepsilon\left(\epsilon-\epsilon^{e}, v\right)_{D^{k}}+\left(\epsilon-\epsilon^{e}, \psi\right)_{D^{k}}-\left(\Delta_{(\alpha-2) / 2}\left(\varphi-\varphi^{e}\right), \psi\right)_{D^{k}} \\
& +\left(\varphi-\varphi^{e}, \phi\right)_{D^{k}}+\left(\partial_{x}\left(\pi-\pi^{e}\right), \partial_{x} \phi\right)_{D^{k}}+\left(\left(\partial_{x}\left(\pi-\pi^{e}\right)\right)^{*}[\phi]+\left\{\partial_{x} \phi\right\}\left[\pi-\pi^{e}\right]\right)_{k+\frac{1}{2}}=0 .
\end{aligned}
$$


We take the test functions

$$
v=\pi, \quad \psi=\epsilon-\varphi, \quad \phi=\pi
$$

we obtain

$$
\begin{aligned}
& \left(\left(\pi-\pi^{e}\right)_{t}, \pi\right)_{D^{k}}-\varepsilon\left(\epsilon-\epsilon^{e}, \pi\right)_{D^{k}}+\left(\epsilon-\epsilon^{e}, \epsilon-\varphi\right)_{D^{k}}-\left(\Delta_{(\alpha-2) / 2}\left(\varphi-\varphi^{e}\right), \epsilon-\varphi\right)_{D^{k}} \\
& +\left(\varphi-\varphi^{e}, \pi\right)_{D^{k}}+\left(\partial_{x}\left(\pi-\pi^{e}\right), \partial_{x} \pi\right)_{D^{k}}+\left(\left(\partial_{x}\left(\pi-\pi^{e}\right)\right)^{*}[\pi]+\left\{\partial_{x} \pi\right\}\left[\pi-\pi^{e}\right]\right)_{k+\frac{1}{2}}=0 .
\end{aligned}
$$

Summing over $k$ and from the admissible condition (4.1) of the numerical flux defined in (3.9), we obtain that

$$
\begin{aligned}
& \left(\pi_{t}, \pi\right)+(\epsilon, \epsilon)+\left(\Delta_{(\alpha-2) / 2} \varphi, \varphi\right)+(1-\gamma)\left\|\partial_{x} \pi\right\|_{L^{2}(\Omega)}^{2}+\mu \sum_{k=1}^{K} \frac{[\pi]_{k+\frac{1}{2}}^{2}}{h} \\
& \leq\left(\pi_{t}^{e}, \pi\right)-\left(\Delta_{(\alpha-2) / 2} \varphi^{e}, \epsilon-\varphi\right)+\left(\Delta_{(\alpha-2) / 2} \varphi, \epsilon\right)+\left(\epsilon^{e}, \epsilon-\varphi\right) \\
& \quad+(\epsilon, \varphi)+\varepsilon\left(\epsilon-\epsilon^{e}, \pi\right)-\left(\varphi-\varphi^{e}, \pi\right)+\left(\partial_{x} \pi^{e}, \partial_{x} \pi\right)+\sum_{k=1}^{K}\left(\left(\partial_{x}\left(\pi^{e}\right)\right)^{*}[\pi]+\left\{\partial_{x} \pi\right\}\left[\pi^{e}\right]\right)_{k+\frac{1}{2}} .
\end{aligned}
$$

Using the definitions of the projections $\mathcal{P}^{+}$in 4.8 , we get

$$
\begin{aligned}
& \left(\pi_{t}, \pi\right)+(\epsilon, \epsilon)+\left(\Delta_{(\alpha-2) / 2} \varphi, \varphi\right)+(1-\gamma)\left\|\partial_{x} \pi\right\|_{L^{2}(\Omega)}^{2}+\mu \sum_{k=1}^{K} \frac{[\pi]_{k+\frac{1}{2}}^{2}}{h} \\
& \leq\left(\pi_{t}^{e}, \pi\right)-\left(\Delta_{(\alpha-2) / 2} \varphi^{e}, \epsilon-\varphi\right)+\left(\Delta_{(\alpha-2) / 2} \varphi, \epsilon\right)+\left(\epsilon^{e}, \epsilon-\varphi\right) \\
& \quad+(\epsilon, \varphi)+\varepsilon\left(\epsilon-\epsilon^{e}, \pi\right)-\left(\varphi-\varphi^{e}, \pi\right) .
\end{aligned}
$$

From the approximation results 4.9 and Young's inequality, we obtain

$$
\begin{aligned}
& \left(\pi_{t}, \pi\right)+(\epsilon, \epsilon)+\left(\Delta_{(\alpha-2) / 2} \varphi, \varphi\right)+(1-\gamma)\left\|\partial_{x} \pi\right\|_{L^{2}(\Omega)}^{2}+\mu \sum_{k=1}^{K} \frac{[\pi]_{k+\frac{1}{2}}^{2}}{h} \\
& \leq c_{3}\|\pi\|_{L^{2}(\Omega)}^{2}+c_{2}\|\varphi\|_{L^{2}(\Omega)}^{2}+c_{1}\|\epsilon\|_{L^{2}(\Omega)}^{2}+C h^{2 N+2} .
\end{aligned}
$$

Recalling Lemma 2.3, we get

$$
\left(\pi_{t}, \pi\right)+(\epsilon, \epsilon)+(1-\gamma)\left\|\partial_{x} \pi\right\|_{L^{2}(\Omega)}^{2}+\mu \sum_{k=1}^{K} \frac{[\pi]_{k+\frac{1}{2}}^{2}}{h} \leq c_{3}\|\pi\|_{L^{2}(\Omega)}^{2}+c_{1}\|\epsilon\|_{L^{2}(\Omega)}^{2}+C h^{2 N+2},
$$

provided $c_{1}$ is sufficiently small such that $c_{1} \leq 1$, we obtain

$$
\left(\pi_{t}, \pi\right)+(1-\gamma)\left\|\partial_{x} \pi\right\|_{L^{2}(\Omega)}^{2}+\mu \sum_{k=1}^{K} \frac{[\pi]_{k+\frac{1}{2}}^{2}}{h} \leq c_{3}\|\pi\|_{L^{2}(\Omega)}^{2}+C h^{2 N+2} .
$$

Employing Gronwall's lemma and standard approximation theory, we can get 4.12 .

For the more general fractional convection-diffusion problem, we introduce a few results and then give the error estimate. 
Lemma 4.2. (see [58]). For any piecewise smooth function $\pi \in L^{2}(\Omega)$, on each cell boundary point we define

$$
\kappa\left(f^{*} ; \pi\right) \equiv \kappa\left(f^{*} ; \pi^{-}, \pi^{+}\right)= \begin{cases}{[w]^{-1}\left(f(\pi)-f^{*}(\pi)\right),} & \text { if }[\pi] \neq 0 \\ \frac{1}{2}\left|f^{\prime}(\bar{\pi})\right|, & \text { if }[\pi]=0\end{cases}
$$

where $f^{*}(\pi) \equiv f^{*}\left(\pi, \pi^{+}\right)$is a monotone numerical flux consistent with the given flux $f$. Then $\kappa\left(f^{*}, \pi\right)$ is nonnegative and bounded for any $\left(\pi, \pi^{+}\right) \in \mathbb{R}$.

To estimate the nonlinear part, we can write it into the following form

$$
\sum_{k=1}^{K} \mathcal{H}_{k}\left(f ; u, u_{h} ; \pi\right)=\sum_{k=1}^{K}\left(f(u)-f\left(u_{h}\right), \frac{\partial}{\partial x} \pi\right)_{D^{k}}+\sum_{k=1}^{K}\left(\left(f(u)-f\left(u_{h}\right)[\pi]\right)_{k+\frac{1}{2}}+\sum_{k=1}^{K}\left(\left(f\left(u_{h}\right)-\hat{f}\right)[\pi]\right)_{k+\frac{1}{2}} .\right.
$$

We can rewrite 4.26 as:

$$
\sum_{k=1}^{K} \mathcal{H}_{k}\left(f ; u, u_{h} ; \pi\right)=\sum_{k=1}^{K}\left(f(u)-f\left(u_{h}\right), \frac{\partial}{\partial x} \pi\right)_{D^{k}}+\sum_{k=1}^{K}\left(\left(f(u)-f\left(\left\{u_{h}\right\}\right)[\pi]\right)_{k+\frac{1}{2}}+\sum_{k=1}^{K}\left(\left(f\left(\left\{u_{h}\right\}\right)-\hat{f}\right)[\pi]\right)_{k+\frac{1}{2}} .\right.
$$

Lemma 4.3. For $\mathcal{H}_{k}\left(f ; u, u_{h} ; \pi\right)$ defined above, we have the following estimate:

$$
\sum_{k=1}^{K} \mathcal{H}_{k}\left(f ; u, u_{h} ; \pi\right) \leq \gamma_{1}\left\|\pi_{x}\right\|_{L^{2}(\Omega)}^{2}+\mu \sum_{k=1}^{K} \frac{[\pi]_{k+\frac{1}{2}}^{2}}{h}+C\|\pi\|_{L^{2}(\Omega)}^{2}\left(1+h^{-1}\|\pi\|_{L^{2}(\Omega)}^{2}\right)+h^{2 N+2} .
$$

Proof. First, we give the estimate of the last term in (4.27), since the exact solution $u$ is continuous on each boundary point, we have that

$$
u_{h}=\left[\pi^{e}\right]-[\pi] .
$$

Employing Young's and the interpolation property [4.10, we can easily show that

$$
\begin{aligned}
\sum_{k=1}^{K}\left(\left(f\left(u_{h}\right)-\hat{f}\right)[\pi]\right)_{k+\frac{1}{2}}= & \sum_{k=1}^{K}\left(\kappa\left(\hat{f} ; u_{h}\right)\left[u_{h}\right][\pi]\right)_{k+\frac{1}{2}}=\sum_{k=1}^{K}\left(\kappa\left(\hat{f} ; u_{h}\right)\left[\pi^{e}\right][\pi]\right)_{k+\frac{1}{2}}-\sum_{k=1}^{K}\left(\kappa\left(\hat{f} ; u_{h}\right)[\pi]^{2}\right)_{k+\frac{1}{2}} \\
& \leq \frac{\mu}{3 h} \sum_{k=1}^{K}[\pi]_{k+\frac{1}{2}}^{2}+\frac{3 h}{4 \mu} \sum_{k=1}^{K}\left[\kappa \pi^{e}\right]_{k+\frac{1}{2}}^{2} \\
& \leq \frac{\mu}{3 h} \sum_{k=1}^{K}[\pi]_{k+\frac{1}{2}}^{2}+C h^{2 N+2} .
\end{aligned}
$$

For the first two terms of the right-hand (4.27), we would like to use the following Taylor expansions

$$
f(u)-f\left(u_{h}\right)=f^{\prime}(u)\left(\pi-\pi^{e}\right)-\frac{1}{2} f_{u}^{\prime \prime}\left(\pi-\pi^{e}\right)^{2},
$$




$$
f(u)-f\left(\left\{u_{h}\right\}\right)=f^{\prime}(u)\left(\{\pi\}-\{\pi\}^{e}\right)-\frac{1}{2} \tilde{f}_{u}^{\prime \prime}\left(\{\pi\}-\{\pi\}^{e}\right)^{2},
$$

where $f_{u}^{\prime \prime}$ and $\tilde{f}_{u}^{\prime \prime}$ are the mean values. These imply the following representation

$$
\sum_{k=1}^{K}\left(f(u)-f\left(u_{h}\right), \frac{\partial}{\partial x} \pi\right)_{D^{k}}+\sum_{k=1}^{K}\left(\left(f(u)-f\left(\left\{u_{h}\right\}\right)[\pi]\right)_{k+\frac{1}{2}}=I+I I+I I I,\right.
$$

where

$$
\begin{aligned}
& \left.I=\sum_{k=1}^{K}\left(f^{\prime}(u) \pi, \frac{\partial}{\partial x} \pi\right)_{D^{k}}+\sum_{k=1}^{K} f^{\prime}(u)\{\pi\}[\pi]\right)_{k+\frac{1}{2}} \\
& \left.I I=-\left(\sum_{k=1}^{K}\left(f^{\prime}(u) \pi^{e}, \frac{\partial}{\partial x} \pi\right)_{D^{k}}+\sum_{k=1}^{K} f^{\prime}(u)\{\pi\}^{e}[\pi]\right)_{k+\frac{1}{2}}\right), \\
& \left.I I I=-\frac{1}{2}\left(\sum_{k=1}^{K}\left(f^{\prime \prime}\left(\pi-\pi^{e}\right)^{2}, \frac{\partial}{\partial x} \pi\right)_{D^{k}}+\sum_{k=1}^{K} \tilde{f}^{\prime \prime}\left(\{\pi\}-\{\pi\}^{e}\right)^{2}[\pi]\right)_{k+\frac{1}{2}}\right),
\end{aligned}
$$

will be estimated separately as below.

For the $I$ term, a simple integration by parts gives

$$
I=-\frac{1}{2} \sum_{k=1}^{K}\left(f^{\prime \prime}(u) \frac{\partial u}{\partial x}, \pi\right)_{D^{k}} \leq C\|\pi\|_{L^{2}(\Omega)}^{2} .
$$

For the $I I$ term, using Young's inequality, we obtain

$$
\begin{aligned}
I I \leq & \frac{\gamma_{1}}{2}\left\|\pi_{x}\right\|_{L^{2}(\Omega)}^{2}+\frac{1}{2 \gamma_{1}}\left\|f^{\prime}(u) \pi^{e}\right\|_{L^{2}(\Omega)}^{2}+\frac{\mu}{3 h} \sum_{k=1}^{K}[\pi]_{k+\frac{1}{2}}^{2}+\frac{3 h}{4 \mu} \sum_{k=1}^{K}\left(f^{\prime}(u)\{\pi\}^{e}\right)_{k+\frac{1}{2}}^{2} \\
& \leq \frac{\gamma_{1}}{2}\left\|\pi_{x}\right\|_{L^{2}(\Omega)}^{2}+\frac{\mu}{3 h} \sum_{k=1}^{K}[\pi]_{k+\frac{1}{2}}^{2}+C h^{2 N+2} .
\end{aligned}
$$

For the $I I I$ term, we use both projection and inverse inequalities, 4.10 and 4.11, to get

$$
\begin{aligned}
I I I \leq & \frac{\gamma_{1}}{2}\left\|\pi_{x}\right\|_{L^{2}(\Omega)}^{2}+\frac{1}{2 \gamma_{1}}\left\|f^{\prime \prime}\left(\pi-\pi^{e}\right)^{2}\right\|_{L^{2}(\Omega)}^{2}+\frac{\mu}{3 h} \sum_{k=1}^{K}[\pi]_{k+\frac{1}{2}}^{2}+\frac{3 h}{4 \mu} \sum_{k=1}^{K}\left(\tilde{f}^{\prime \prime}\left\{\pi-\pi^{e}\right\}^{2}\right)_{k+\frac{1}{2}}^{2} \\
& \leq \frac{\gamma_{1}}{2}\left\|\pi_{x}\right\|_{L^{2}(\Omega)}^{2}+\frac{\mu}{3 h} \sum_{k=1}^{K}[\pi]_{k+\frac{1}{2}}^{2}+C\left\|\pi-\pi^{e}\right\|_{\infty}^{2}\left(\|\pi\|_{L^{2}(\Omega)}^{2}+\left\|\pi^{e}\right\|_{L^{2}(\Omega)}^{2}+h\|\pi\|_{\Gamma_{h}}^{2}+h\left\|\pi^{e}\right\|_{\Gamma_{h}}^{2}\right) \\
& \leq \frac{\gamma_{1}}{2}\left\|\pi_{x}\right\|_{L^{2}(\Omega)}^{2}+\frac{\mu}{3 h} \sum_{k=1}^{K}[\pi]_{k+\frac{1}{2}}^{2}+C\left(\|\pi\|_{L^{2}(\Omega)}^{2}+h^{2 N+2}\right)\left(1+\left\|\pi-\pi^{e}\right\|_{\infty}^{2}\right) .
\end{aligned}
$$

Using the approximation results in 4.10 and 4.11), we have

$$
\begin{aligned}
I I I \leq & \frac{\gamma_{1}}{2}\left\|\pi_{x}\right\|_{L^{2}(\Omega)}^{2}+\frac{\mu}{3 h} \sum_{k=1}^{K}[\pi]_{k+\frac{1}{2}}^{2}+C\left(\|\pi\|_{L^{2}(\Omega)}^{2}+h^{2 N+2}\right)\left(1+h^{-1}\|\pi\|_{L^{2}(\Omega)}^{2}+h^{2 N+1}\right) \\
& \leq \frac{\gamma_{1}}{2}\left\|\pi_{x}\right\|_{L^{2}(\Omega)}^{2}+\frac{\mu}{3 h} \sum_{k=1}^{K}[\pi]_{k+\frac{1}{2}}^{2}+C\|\pi\|_{L^{2}\left(\Omega_{h}\right)}^{2}\left(1+h^{-1}\|\pi\|_{L^{2}(\Omega)}^{2}\right)+h^{2 N+2} .
\end{aligned}
$$


Combining (4.30), 4.37), 4.38, 4.40, and 4.27), we obtain

$$
\sum_{k=1}^{K} \mathcal{H}_{k}\left(f ; u, u_{h} ; \pi\right) \leq \gamma_{1}\left\|\pi_{x}\right\|_{L^{2}(\Omega)}^{2}+\frac{\mu}{h} \sum_{k=1}^{K}[\pi]_{k+\frac{1}{2}}^{2}+C\|\pi\|_{L^{2}(\Omega)}^{2}\left(1+h^{-1}\|\pi\|_{L^{2}(\Omega)}^{2}\right)+h^{2 N+2} .
$$

Theorem 4.3. Let $u$ be the exact solution of the problem (1.1), and let $u_{h}$ be the numerical solution of the semi-discrete DDG scheme 3.10. Then for small enough $h$, we have the following error estimates:

$$
\left\|u-u_{h}\right\|_{L^{2}(\Omega)} \leq C h^{N+1},
$$

where the constant $C$ is dependent upon $T$ and some norms of the solutions.

Proof. The exact solution of the above (4.13) satisfies

$$
\begin{aligned}
& \left(u_{t}, v\right)_{D^{k}}-\varepsilon(p, v)_{D^{k}}+(p, \psi)_{D^{k}}-\left(\Delta_{(\alpha-2) / 2} q, \psi\right)_{D^{k}}+(q, \phi)_{D^{k}}-\left(f(u), \partial_{x} v\right)_{D^{k}}+\left(\hat{n} \cdot f(u)^{*}, v\right)_{\partial D^{k}} \\
& \quad+\left(\partial_{x} u, \partial_{x} \phi\right)_{D^{k}}+\left(\left(\partial_{x} u\right)^{*}[\phi]+\left\{\partial_{x} \phi\right\}[u]\right)_{k+\frac{1}{2}}=0 .
\end{aligned}
$$

Subtracting 4.43) from (3.10), we have the following error equation

$$
\begin{aligned}
& \left(\left(u-u_{h}\right)_{t}, v\right)_{D^{k}}-\varepsilon\left(p-p_{h}, v\right)_{D^{k}}+\left(p-p_{h}, \psi\right)_{D^{k}}-\left(\Delta_{(\alpha-2) / 2}\left(q-q_{h}\right), \psi\right)_{D^{k}}-\left(f(u)-f\left(u_{h}\right), \partial_{x} v\right)_{D^{k}} \\
& +\left(\hat{n} \cdot\left(f(u)-f\left(u_{h}\right)\right)^{*}, v\right)_{\partial D^{k}}+\left(q-q_{h}, \phi\right)_{D^{k}}+\left(\partial_{x}\left(u-u_{h}\right), \partial_{x} \phi\right)_{D^{k}}+\left(\left(\partial_{x}\left(u-u_{h}\right)\right)^{*}[\phi]+\left\{\partial_{x} \phi\right\}\left[u-u_{h}\right]\right)_{k+\frac{1}{2}}=0 .
\end{aligned}
$$

From 4.27), we can rewrite (4.44) as:

$$
\begin{aligned}
& \left(\left(u-u_{h}\right)_{t}, v\right)_{D^{k}}-\varepsilon\left(p-p_{h}, v\right)_{D^{k}}+\left(p-p_{h}, \psi\right)_{D^{k}}-\left(\Delta_{(\alpha-2) / 2}\left(q-q_{h}\right), \psi\right)_{D^{k}} \\
& -\mathcal{H}_{k}\left(f ; u, u_{h} ; v\right)+\left(q-q_{h}, \phi\right)_{D^{k}}+\left(\partial_{x}\left(u-u_{h}\right), \partial_{x} \phi\right)_{D^{k}}+\left(\left(\partial_{x}\left(u-u_{h}\right)\right)^{*}[\phi]+\left\{\partial_{x} \phi\right\}\left[u-u_{h}\right]\right)_{k+\frac{1}{2}}=0 .
\end{aligned}
$$

From the Galerkin orthogonality 4.45], we get

$$
\begin{aligned}
& \left(\left(\pi-\pi^{e}\right)_{t}, v\right)_{D^{k}}-\varepsilon\left(\epsilon-\epsilon^{e}, v\right)_{D^{k}}+\left(\epsilon-\epsilon^{e}, \psi\right)_{D^{k}}-\left(\Delta_{(\alpha-2) / 2}\left(\varphi-\varphi^{e}\right), \psi\right)_{D^{k}}-\mathcal{H}_{k}\left(f ; u, u_{h} ; v\right) \\
& +\left(\varphi-\varphi^{e}, \phi\right)_{D^{k}}+\left(\partial_{x}\left(\pi-\pi^{e}\right), \partial_{x} \phi\right)_{D^{k}}+\left(\left(\partial_{x}\left(\pi-\pi^{e}\right)\right)^{*}[\phi]+\left\{\partial_{x} \phi\right\}\left[\pi-\pi^{e}\right]\right)_{k+\frac{1}{2}}=0 .
\end{aligned}
$$

Following the proof of Theorem 4.3 , we set

$$
v=\pi, \quad \psi=\epsilon-\varphi, \quad \phi=\pi .
$$

Summing over $k$ and from the admissible condition 4.1, definitions of the projections $\mathcal{P}^{+}$, the approximation 
results 4.9 and Young's inequality, we derive the following inequality:

$$
\begin{aligned}
& \left(\pi_{t}, \pi\right)+(\epsilon, \epsilon)+\left(\Delta_{(\alpha-2) / 2} \varphi, \varphi\right)+(1-\gamma)\left\|\partial_{x} \pi\right\|_{L^{2}(\Omega)}^{2}+\mu \sum_{k=1}^{K} \frac{[\pi]_{k+\frac{1}{2}}^{2}}{h} \\
& \leq c_{3}\|\pi\|_{L^{2}(\Omega)}^{2}+c_{2}\|\varphi\|_{L^{2}(\Omega)}^{2}+c_{1}\|\epsilon\|_{L^{2}(\Omega)}^{2}+C h^{2 N+2}+\sum_{k=1}^{K} \mathcal{H}_{k}\left(f ; u, u_{h} ; \pi\right) .
\end{aligned}
$$

Recalling Lemmas 2.3 and 4.3 , we get

$$
\left(\pi_{t}, \pi\right)+(\epsilon, \epsilon) \leq c_{3}\|\pi\|_{L^{2}(\Omega)}^{2}+c_{1}\|\epsilon\|_{L^{2}(\Omega)}^{2}+C h^{2 N+2}+C\|\pi\|_{L^{2}(\Omega)}^{2}\left(1+h^{-1}\|\pi\|_{L^{2}(\Omega)}^{2}+h^{2 N+2}\right),
$$

provided $c_{1}$ is sufficiently small such that $c_{1} \leq 1$, we obtain

$$
\left(\pi_{t}, \pi\right) \leq c_{3}\|\pi\|_{L^{2}(\Omega)}^{2}+C\|\pi\|_{L^{2}(\Omega)}^{2}\left(1+h^{-1}\|\pi\|_{L^{2}(\Omega)}^{2}\right)+h^{2 N+2} .
$$

From the Gronwall's lemma and standard approximation theory, the desired result follows.

\section{DDG method for nonlinear fractional Schrödinger equation}

In this section, we present and analyze a direct discontinuous Galerkin method for the following:

$$
\begin{aligned}
& i \frac{\partial u}{\partial t}-\varepsilon_{1}(-\Delta)^{\frac{\alpha}{2}} u+\varepsilon_{2} f\left(|u|^{2}\right) u=0, \quad x \in \mathbb{R}, t \in(0, T], \\
& u(x, 0)=u_{0}(x), \quad x \in \mathbb{R},
\end{aligned}
$$

We introduce the auxiliary variables $p, q$ and set

$$
p=\Delta_{(\alpha-2) / 2} q, \quad q=\frac{\partial^{2}}{\partial x^{2}} u
$$

then, the nonlinear fractional Schrödinger problem can be rewritten as

$$
\begin{aligned}
& i \frac{\partial u}{\partial t}+\varepsilon_{1} e+\varepsilon_{2} f\left(|u|^{2}\right) u=0, \\
& r=\Delta_{(\alpha-2) / 2} q, \quad s=\frac{\partial^{2}}{\partial x^{2}} u .
\end{aligned}
$$

For actual numerical implementation, it might be more efficient if we decompose the complex function $u(x, t)$ into its real and imaginary parts by writing

$$
u(x, t)=p(x, t)+i q(x, t),
$$

where $p, q$ are real functions. Under the new notation, the problem 5.3 can be written as

$$
\begin{aligned}
& \frac{\partial p}{\partial t}+\varepsilon_{1} e+\varepsilon_{2} f\left(p^{2}+q^{2}\right) q=0, \\
& e=\Delta_{(\alpha-2) / 2} r, \quad r=\frac{\partial^{2}}{\partial x^{2}} q, \\
& \frac{\partial q}{\partial t}-\varepsilon_{1} l-\varepsilon_{2} f\left(p^{2}+q^{2}\right) p=0, \\
& l=\Delta_{(\alpha-2) / 2} w, \quad w=\frac{\partial^{2}}{\partial x^{2}} p .
\end{aligned}
$$


We now apply the direct discontinuous Galerkin discretization to (5.5): find $p_{h}, q_{h}, e_{h}, l_{h}, r_{h}, w_{h} \in V_{k}^{N}$, such that for all test functions $\vartheta, \varphi, \phi, \chi, \zeta, \psi \in V_{k}^{N}$,

$$
\begin{aligned}
& \left(\left(p_{h}\right)_{t}, \vartheta\right)_{D^{k}}+\varepsilon_{1}\left(e_{h}, \vartheta\right)_{D^{k}}+\varepsilon_{2}\left(f\left(p_{h}^{2}+q_{h}^{2}\right) q_{h}, \vartheta\right)_{D^{k}}=0 \\
& \left(e_{h}, \varphi\right)_{D^{k}}=\left(\Delta_{(\alpha-2) / 2} r_{h}, \varphi\right)_{D^{k}} \\
& \left(r_{h}, \phi\right)_{D^{k}}=-\left(\partial_{x} q_{h}, \partial_{x} \phi\right)_{D^{k}}-\left(\left(\partial_{x} q_{h}\right)^{*}[\phi]+\left\{\partial_{x} \phi\right\}\left[q_{h}\right]\right)_{k+\frac{1}{2}} \\
& \left(\left(q_{h}\right)_{t}, \chi\right)_{D^{k}}-\varepsilon_{1}\left(l_{h}, \chi\right)_{D^{k}}-\varepsilon_{2}\left(f\left(p_{h}^{2}+q_{h}^{2}\right) p_{h}, \chi\right)_{D^{k}}=0 \\
& \left(l_{h}, \zeta\right)_{D^{k}}=\left(\Delta_{(\alpha-2) / 2} w_{h}, \zeta\right)_{D^{k}} \\
& \left(w_{h}, \psi\right)_{D^{k}}=-\left(\partial_{x} p_{h}, \partial_{x} \psi\right)_{D^{k}}-\left(\left(\partial_{x} p_{h}\right)^{*}[\psi]+\left\{\partial_{x} \psi\right\}\left[p_{h}\right]\right)_{k+\frac{1}{2}}
\end{aligned}
$$

\section{Stability and error estimates}

In the following we discuss stability and accuracy of the proposed scheme, for the nonlinear fractional Schrödinger problem.

\subsection{Stability analysis}

In order to carry out the analysis of the DDG scheme, we have the following results.

Theorem 6.1. ( $L^{2}$ stability). The solution to the scheme (5.6) satisfies the $\left\|u_{h}(x, T)\right\|_{L^{2}(\Omega)} \leq c\left\|u_{0}(x)\right\|_{L^{2}(\Omega)}$ for any $T>0$.

Proof. Set $(\vartheta, \varphi, \phi, \chi, \zeta, \psi)=\left(p_{h}, e_{h}-r_{h}, q_{h}, q_{h}, l_{h}-w_{h}, p_{h}\right)$ in (5.6), we get

$$
\begin{aligned}
& \left(\left(p_{h}\right)_{t}, p_{h}\right)_{D^{k}}+\left(\left(q_{h}\right)_{t}, q_{h}\right)_{D^{k}}+\left(e_{h}, e_{h}\right)_{D^{k}}+\left(l_{h}, l_{h}\right)_{D^{k}}+\left(\Delta_{(\alpha-2) / 2} w_{h}, w_{h}\right)_{D^{k}}+\left(\Delta_{(\alpha-2) / 2} r_{h}, r_{h}\right)_{D^{k}} \\
& =\left(\Delta_{(\alpha-2) / 2} w_{h}, l_{h}\right)_{D^{k}}+\left(\Delta_{(\alpha-2) / 2} r_{h}, e_{h}\right)_{D^{k}}-\left(r_{h}, q_{h}\right)_{D^{k}}-\left(w_{h}, p_{h}\right)_{D^{k}}+\left(e_{h}, r_{h}\right)_{D^{k}}+\left(l_{h}, w_{h}\right)_{D^{k}} \\
& \quad-\varepsilon_{1}\left(e_{h}, p_{h}\right)_{D^{k}}+\varepsilon_{1}\left(l_{h}, q_{h}\right)_{D^{k}}-\left(\partial_{x} q_{h}, \partial_{x} \phi\right)_{D^{k}}-\left(\left(\partial_{x} q_{h}\right)^{*}[\phi]+\left\{\partial_{x} \phi\right\}\left[q_{h}\right]\right)_{k+\frac{1}{2}}-\left(\partial_{x} p_{h}, \partial_{x} \psi\right)_{D^{k}} \\
& \quad-\left(\left(\partial_{x} p_{h}\right)^{*}[\psi]+\left\{\partial_{x} \psi\right\}\left[p_{h}\right]\right)_{k+\frac{1}{2}} .
\end{aligned}
$$

Summing over $k$ and from the admissible condition 4.1 of the numerical flux defined in (3.9), we obtain that

$$
\begin{aligned}
& \left(\left(p_{h}\right)_{t}, p_{h}\right)+\left(\left(q_{h}\right)_{t}, q_{h}\right)+\left(e_{h}, e_{h}\right)+\left(l_{h}, l_{h}\right)+\left(\Delta_{(\alpha-2) / 2} w_{h}, w_{h}\right)+\left(\Delta_{(\alpha-2) / 2} r_{h}, r_{h}\right) \\
& +\left(1-\gamma_{2}\right)\left\|\partial_{x} q_{h}\right\|_{L^{2}(\Omega)}^{2}+\mu_{2} \sum_{k=1}^{K} \frac{\left[q_{h}\right]_{k+\frac{1}{2}}^{2}}{h}+\left(1-\gamma_{1}\right)\left\|\partial_{x} p_{h}\right\|_{L^{2}(\Omega)}^{2}+\mu_{1} \sum_{k=1}^{K} \frac{\left[p_{h}\right]_{k+\frac{1}{2}}^{2}}{h} \\
& \leq\left(\Delta_{(\alpha-2) / 2} w_{h}, l_{h}\right)+\left(\Delta_{(\alpha-2) / 2} r_{h}, e_{h}\right)-\left(r_{h}, q_{h}\right)_{D^{k}}-\left(w_{h}, p_{h}\right)+\left(e_{h}, r_{h}\right)+\left(l_{h}, w_{h}\right) \\
& \quad-\varepsilon_{1}\left(e_{h}, p_{h}\right)+\varepsilon_{1}\left(l_{h}, q_{h}\right)
\end{aligned}
$$


Employing Young's inequality and Lemma 2.5, we obtain

$$
\begin{aligned}
& \left(\left(p_{h}\right)_{t}, p_{h}\right)+\left(\left(q_{h}\right)_{t}, q_{h}\right)+\left(e_{h}, e_{h}\right)+\left(l_{h}, l_{h}\right)+\left(\Delta_{(\alpha-2) / 2} w_{h}, w_{h}\right)+\left(\Delta_{(\alpha-2) / 2} r_{h}, r_{h}\right) \\
& +\left(1-\gamma_{2}\right)\left\|\partial_{x} q_{h}\right\|_{L^{2}(\Omega)}^{2}+\mu_{2} \sum_{k=1}^{K} \frac{\left[q_{h}\right]_{k+\frac{1}{2}}^{2}}{h}+\left(1-\gamma_{1}\right)\left\|\partial_{x} p_{h}\right\|_{L^{2}(\Omega)}^{2}+\mu_{1} \sum_{k=1}^{K} \frac{\left[p_{h}\right]_{k+\frac{1}{2}}^{2}}{h} \\
& \leq c_{4}\left\|w_{h}\right\|_{L^{2}(\Omega)}^{2}+c_{3}\left\|r_{h}\right\|_{L^{2}(\Omega)}^{2}+c_{2}\left\|e_{h}\right\|_{L^{2}(\Omega)}^{2}+c_{1}\left\|l_{h}\right\|_{L^{2}(\Omega)}^{2}+c_{5}\left\|p_{h}\right\|_{L^{2}(\Omega)}^{2}+c_{6}\left\|q_{h}\right\|_{L^{2}(\Omega)}^{2} .
\end{aligned}
$$

Recalling Lemma 2.3 , provided $c_{i}, i=1,2$ are sufficiently small such that $c_{i} \leq 1$, we obtain

$$
\begin{aligned}
& \left(\left(p_{h}\right)_{t}, p_{h}\right)+\left(\left(q_{h}\right)_{t}, q_{h}\right)+\left(1-\gamma_{2}\right)\left\|\partial_{x} q_{h}\right\|_{L^{2}(\Omega)}^{2}+\mu_{2} \sum_{k=1}^{K} \frac{\left[q_{h}\right]_{k+\frac{1}{2}}^{2}}{h}+\left(1-\gamma_{1}\right)\left\|\partial_{x} p_{h}\right\|_{L^{2}(\Omega)}^{2}+\mu_{1} \sum_{k=1}^{K} \frac{\left[p_{h}\right]_{k+\frac{1}{2}}^{2}}{h} \\
& \leq c_{5}\left\|p_{h}\right\|_{L^{2}(\Omega)}^{2}+c_{6}\left\|q_{h}\right\|_{L^{2}(\Omega)}^{2} .
\end{aligned}
$$

Employing Gronwall's lemma, we obtain $\left\|u_{h}(x, T)\right\|_{L^{2}(\Omega)} \leq c\left\|u_{0}(x)\right\|_{L^{2}(\Omega)}$.

\subsection{Error estimates}

We consider the linear fractional Schrödinger equation

$$
i \frac{\partial u}{\partial t}-\varepsilon_{1}(-\Delta)^{\frac{\alpha}{2}} u+\varepsilon_{2} u=0 .
$$

It is easy to verify that the exact solution of the above (6.5) satisfies

$$
\begin{aligned}
& \left(p_{t}, \vartheta\right)_{D^{k}}+\varepsilon_{1}(e, \vartheta)_{D^{k}}+\varepsilon_{2}(q, \vartheta)_{D^{k}}=0 \\
& (e, \varphi)_{D^{k}}=\left(\Delta_{(\alpha-2) / 2} r, \varphi\right)_{D^{k}}, \\
& (r, \phi)_{D^{k}}=-\left(\partial_{x} q, \partial_{x} \phi\right)_{D^{k}}-\left(\left(\partial_{x} q\right)^{*}[\phi]+\left\{\partial_{x} \phi\right\}[q]\right)_{k+\frac{1}{2}}, \\
& \left(q_{t}, \chi\right)_{D^{k}}-\varepsilon_{1}(l, \chi)_{D^{k}}-\varepsilon_{2}(p, \chi)_{D^{k}}=0, \\
& (l, \zeta)_{D^{k}}=\left(\Delta_{(\alpha-2) / 2} w, \zeta\right)_{D^{k}}, \\
& (w, \psi)_{D^{k}}=-\left(\partial_{x} p, \partial_{x} \psi\right)_{D^{k}}-\left(\left(\partial_{x} p\right)^{*}[\psi]+\left\{\partial_{x} \psi\right\}[p]\right)_{k+\frac{1}{2}}
\end{aligned}
$$

Subtracting (6.6), from the linear fractional Schrödinger equation (5.6), we have the following error equation

$$
\begin{aligned}
& \left(\left(p-p_{h}\right)_{t}, \vartheta\right)_{D^{k}}+\left(\left(q-q_{h}\right)_{t}, \chi\right)_{D^{k}}-\left(\Delta_{(\alpha-2) / 2}\left(r-r_{h}\right), \varphi\right)_{D^{k}}-\left(\Delta_{(\alpha-2) / 2}\left(w-w_{h}\right), \zeta\right)_{D^{k}}+\left(\left(q-q_{h}\right)_{x}, \phi_{x}\right)_{D^{k}} \\
& +\left(\left(p-p_{h}\right)_{x}, \psi_{x}\right)_{D^{k}}+\varepsilon_{2}\left(q-q_{h}, \vartheta\right)_{D^{k}}-\varepsilon_{2}\left(p-p_{h}, \chi\right)_{D^{k}}+\left(r-r_{h}, \phi\right)_{D^{k}}+\left(l-l_{h}, \zeta\right)_{D^{k}}+\left(e-e_{h}, \varphi\right)_{D^{k}} \\
& +\left(w-w_{h}, \psi\right)_{D^{k}}-\varepsilon_{1}\left(l-l_{h}, \chi\right)_{D^{k}}+\varepsilon_{1}\left(e-e_{h}, \vartheta\right)_{D^{k}}+\left(\left(\partial_{x}\left(p-p_{h}\right)\right)^{*}[\psi]+\left\{\partial_{x} \psi\right\}\left[p-p_{h}\right]\right)_{k+\frac{1}{2}} \\
& +\left(\left(\partial_{x}\left(q-q_{h}\right)\right)^{*}[\phi]+\left\{\partial_{x} \phi\right\}\left[q-q_{h}\right]\right)_{k+\frac{1}{2}}=0 .
\end{aligned}
$$


Theorem 6.2. Let $u$ be the exact solution of the problem (6.5), and let $u_{h}$ be the numerical solution of the semi-discrete DDG scheme (5.6). Then for small enough $h$, we have the following error estimates:

$$
\left\|u(., t)-u_{h}(., t)\right\|_{L^{2}\left(\Omega_{h}\right)} \leq C h^{N+1},
$$

where the constant $C$ is dependent upon $T$ and some norms of the solutions.

\section{Proof. Let}

$$
\begin{aligned}
& \pi=\mathcal{P}^{+} p-p_{h}, \quad \pi^{e}=\mathcal{P}^{+} p-p, \quad \epsilon=\mathcal{P}^{+} r-r_{h}, \quad \epsilon^{e}=\mathcal{P}^{+} r-r, \quad \phi_{1}=\mathcal{P}^{+} e-e_{h}, \quad \phi_{1}^{e}=\mathcal{P}^{+} e-e, \\
& \sigma=\mathcal{P}^{+} q-q_{h}, \quad \sigma^{e}=\mathcal{P}^{+} q-q, \quad \phi_{2}=\mathcal{P}^{+} l-l_{h}, \quad \phi_{2}^{e}=\mathcal{P}^{+} l-l, \quad \varphi_{1}=\mathcal{P}^{+} w-w_{h}, \quad \varphi_{1}^{e}=\mathcal{P}^{+} w-w .
\end{aligned}
$$

From the Galerkin orthogonality 6.15$)$, we get

$$
\begin{aligned}
& \left(\left(\pi-\pi^{e}\right)_{t}, \vartheta\right)_{D^{k}}+\left(\left(\sigma-\sigma^{e}\right)_{t}, \chi\right)_{D^{k}}-\left(\Delta_{(\alpha-2) / 2}\left(\epsilon-\epsilon^{e}\right), \varphi\right)_{D^{k}}-\left(\Delta_{(\alpha-2) / 2}\left(\varphi_{1}-\varphi_{1}^{e}\right), \zeta\right)_{D^{k}}+\left(\left(\sigma-\sigma^{e}\right)_{x}, \phi_{x}\right)_{D^{k}} \\
& +\left(\left(\pi-\pi^{e}\right)_{x}, \psi_{x}\right)_{D^{k}}+\varepsilon_{2}\left(\sigma-\sigma^{e}, \vartheta\right)_{D^{k}}-\varepsilon_{2}\left(\pi-\pi^{e}, \chi\right)_{D^{k}}+\left(\epsilon-\epsilon^{e}, \phi\right)_{D^{k}}+\left(\phi_{2}-\phi_{2}^{e}, \zeta\right)_{D^{k}}+\left(\phi_{1}-\phi_{1}^{e}, \varphi\right)_{D^{k}} \\
& +\left(\varphi_{1}-\varphi_{1}^{e}, \psi\right)_{D^{k}}-\varepsilon_{1}\left(\phi_{2}-\phi_{2}^{e}, \chi\right)_{D^{k}}+\varepsilon_{1}\left(\phi_{1}-\phi_{1}^{e}, \vartheta\right)_{D^{k}}+\left(\left(\partial_{x}\left(\pi-\pi^{e}\right)\right)^{*}[\psi]+\left\{\partial_{x} \psi\right\}\left[\pi-\pi^{e}\right]\right)_{k+\frac{1}{2}} \\
& \left(\left(\partial_{x}\left(\sigma-\sigma^{e}\right)\right)^{*}[\phi]+\left\{\partial_{x} \phi\right\}\left[\sigma-\sigma^{e}\right]\right)_{k+\frac{1}{2}}=0 .
\end{aligned}
$$

We take the test functions

$$
\vartheta=\pi, \quad \varphi=\phi_{1}-\epsilon, \quad \phi=\sigma, \quad \chi=\sigma, \quad \zeta=\phi_{2}-\varphi, \quad \psi=\pi,
$$

we obtain

$$
\begin{aligned}
& \left(\left(\pi-\pi^{e}\right)_{t}, \pi\right)_{D^{k}}+\left(\left(\sigma-\sigma^{e}\right)_{t}, \sigma\right)_{D^{k}}-\left(\Delta_{(\alpha-2) / 2}\left(\epsilon-\epsilon^{e}\right), \phi_{1}-\epsilon\right)_{D^{k}}-\left(\Delta_{(\alpha-2) / 2}\left(\varphi_{1}-\varphi_{1}^{e}\right), \phi_{2}-\varphi_{1}\right)_{D^{k}} \\
& +\left(\left(\sigma-\sigma^{e}\right)_{x}, \sigma_{x}\right)_{D^{k}}+\left(\left(\pi-\pi^{e}\right)_{x}, \pi_{x}\right)_{D^{k}}+\varepsilon_{2}\left(\sigma-\sigma^{e}, \pi\right)_{D^{k}}-\varepsilon_{2}\left(\pi-\pi^{e}, \sigma\right)_{D^{k}}+\left(\epsilon-\epsilon^{e}, \sigma\right)_{D^{k}} \\
& +\left(\phi_{2}-\phi_{2}^{e}, \phi_{2}-\varphi_{1}\right)_{D^{k}}+\left(\phi_{1}-\phi_{1}^{e}, \phi_{1}-\epsilon\right)_{D^{k}}+\left(\varphi_{1}-\varphi_{1}^{e}, \pi\right)_{D^{k}}-\varepsilon_{1}\left(\phi_{2}-\phi_{2}^{e}, \sigma\right)_{D^{k}}+\varepsilon_{1}\left(\phi_{1}-\phi_{1}^{e}, \pi\right)_{D^{k}} \\
& +\left(\left(\partial_{x}\left(\pi-\pi^{e}\right)\right)^{*}[\pi]+\left\{\partial_{x} \pi\right\}\left[\pi-\pi^{e}\right]\right)_{k+\frac{1}{2}}+\left(\left(\partial_{x}\left(\sigma-\sigma^{e}\right)\right)^{*}[\sigma]+\left\{\partial_{x} \sigma\right\}\left[\sigma-\sigma^{e}\right]\right)_{k+\frac{1}{2}}=0 .
\end{aligned}
$$

Summing over $k$ and from the admissible condition 4.1 of the numerical flux defined in $(3.9)$, we obtain that

$$
\begin{aligned}
& \left(\pi_{t}, \pi\right)+\left(\sigma_{t}, \sigma\right)+\left(\Delta_{(\alpha-2) / 2} \epsilon, \epsilon\right)+\left(\Delta_{(\alpha-2) / 2} \varphi_{1}, \varphi_{1}\right)+\left(1-\gamma_{2}\right)\left\|\partial_{x} \sigma_{h}\right\|_{L^{2}(\Omega)}^{2} \\
& +\mu_{2} \sum_{k=1}^{K} \frac{\left[\sigma_{h}\right]_{k+\frac{1}{2}}^{2}}{h}+\left(1-\gamma_{1}\right)\left\|\partial_{x} \pi_{h}\right\|_{L^{2}(\Omega)}^{2}+\mu_{1} \sum_{k=1}^{K} \frac{\left[\pi_{h}\right]_{k+\frac{1}{2}}^{2}}{h}+\left(\phi_{2}, \phi_{2}\right)+\left(\phi_{1}, \phi_{1}\right) \\
& \leq\left(\pi_{t}^{e}, \pi\right)+\left(\sigma_{t}^{e}, \sigma\right)-\left(\Delta_{(\alpha-2) / 2} \epsilon^{e}, \phi_{1}-\epsilon\right)+\left(\Delta_{(\alpha-2) / 2} \epsilon, \phi_{1}\right)-\left(\Delta_{(\alpha-2) / 2} \varphi_{1}^{e}, \phi_{2}-\varphi_{1}\right) \\
& \quad+\left(\Delta_{(\alpha-2) / 2} \varphi_{1}, \phi_{2}\right)+\left(\sigma_{x}^{e}, \sigma_{x}\right)+\left(\pi_{x}^{e}, \pi_{x}\right)-\varepsilon_{2}\left(\sigma^{e}, \pi\right)-\varepsilon_{2}\left(\pi^{e}, \sigma\right)-\left(\epsilon-\epsilon^{e}, \sigma\right) \\
& \quad+\left(\phi_{2}, \varphi_{1}\right)+\left(\phi_{1}, \epsilon\right)+\left(\phi_{2}^{e}, \phi_{2}-\varphi_{1}\right)+\left(\phi_{1}^{e}, \phi_{1}-\epsilon\right)+\varepsilon_{1}\left(\phi_{2}-\phi_{2}^{e}, \sigma\right)-\varepsilon_{1}\left(\phi_{1}-\phi_{1}^{e}, \pi\right) \\
& \quad-\left(\varphi_{1}-\varphi_{1}^{e}, \pi\right)+\sum_{k=1}^{K}\left(\left(\left(\partial_{x}\left(\pi^{e}\right)\right)^{*}[\pi]+\left\{\partial_{x} \pi\right\}\left[\pi^{e}\right]\right)_{k+\frac{1}{2}}+\left(\left(\partial_{x}\left(\sigma^{e}\right)\right)^{*}[\sigma]+\left\{\partial_{x} \sigma\right\}\left[\sigma^{e}\right]\right)_{k+\frac{1}{2}}\right)
\end{aligned}
$$


Using the definitions of the projections $\mathcal{P}^{+}$in 4.8 , we get

$$
\begin{aligned}
& \left(\pi_{t}, \pi\right)+\left(\sigma_{t}, \sigma\right)+\left(\Delta_{(\alpha-2) / 2} \epsilon, \epsilon\right)+\left(\Delta_{(\alpha-2) / 2} \varphi_{1}, \varphi_{1}\right)+\left(1-\gamma_{2}\right)\left\|\partial_{x} \sigma_{h}\right\|_{L^{2}(\Omega)}^{2} \\
& +\mu_{2} \sum_{k=1}^{K} \frac{\left[\sigma_{h}\right]_{k+\frac{1}{2}}^{2}}{h}+\left(1-\gamma_{1}\right)\left\|\partial_{x} \pi_{h}\right\|_{L^{2}(\Omega)}^{2}+\mu_{1} \sum_{k=1}^{K} \frac{\left[\pi_{h}\right]_{k+\frac{1}{2}}^{2}}{h}+\left(\phi_{2}, \phi_{2}\right)+\left(\phi_{1}, \phi_{1}\right) \\
& \leq\left(\pi_{t}^{e}, \pi\right)+\left(\sigma_{t}^{e}, \sigma\right)-\left(\Delta_{(\alpha-2) / 2} \epsilon^{e}, \phi_{1}-\epsilon\right)+\left(\Delta_{(\alpha-2) / 2} \epsilon, \phi_{1}\right)-\left(\Delta_{(\alpha-2) / 2} \varphi_{1}^{e}, \phi_{2}-\varphi_{1}\right) \\
& \quad+\left(\Delta_{(\alpha-2) / 2} \varphi_{1}, \phi_{2}\right)-\varepsilon_{2}\left(\sigma^{e}, \pi\right)-\varepsilon_{2}\left(\pi^{e}, \sigma\right)-\left(\epsilon-\epsilon^{e}, \sigma\right)+\left(\phi_{2}, \varphi_{1}\right) \\
& \quad+\left(\phi_{1}, \epsilon\right)+\left(\phi_{2}^{e}, \phi_{2}-\varphi_{1}\right)+\left(\phi_{1}^{e}, \phi_{1}-\epsilon\right)+\varepsilon_{1}\left(\phi_{2}-\phi_{2}^{e}, \sigma\right)-\varepsilon_{1}\left(\phi_{1}-\phi_{1}^{e}, \pi\right)-\left(\varphi_{1}-\varphi_{1}^{e}, \pi\right)
\end{aligned}
$$

From the approximation results 4.9 , Young's inequality and recalling Lemma 2.3 , we obtain

$$
\begin{aligned}
& \left(\pi_{t}, \pi\right)_{D^{k}}+\left(\sigma_{t}, \sigma\right)+\left(1-\gamma_{2}\right)\left\|\partial_{x} \sigma_{h}\right\|_{L^{2}(\Omega)}^{2}+\mu_{2} \sum_{k=1}^{K} \frac{\left[\sigma_{h}\right]_{k+\frac{1}{2}}^{2}}{h}+\left(1-\gamma_{1}\right)\left\|\partial_{x} \pi_{h}\right\|_{L^{2}(\Omega)}^{2}+\mu_{1} \sum_{k=1}^{K} \frac{\left[\pi_{h}\right]_{k+\frac{1}{2}}^{2}}{h} \\
& +\left(\phi_{2}, \phi_{2}\right)+\left(\phi_{1}, \phi_{1}\right)_{D^{k}} \leq c_{1}\left\|\phi_{1}\right\|_{L^{2}(\Omega)}^{2}+c_{1}\left\|\phi_{2}\right\|_{L^{2}(\Omega)}^{2}+c_{3}\|\pi\|_{L^{2}(\Omega)}^{2}+c_{4}\|\sigma\|_{L^{2}(\Omega)}^{2}
\end{aligned}
$$

provided $c_{i}, i=1,2$ are sufficiently small such that $c_{i} \leq 1$, employing Gronwall's lemma and standard approximation theory, we can get 6.8$)$.

\section{DDG method for strongly nonlinear coupled fractional Schrödinger equations}

In this section, we present DDG method for the strongly coupled nonlinear fractional Schrödinger equations

$$
\begin{aligned}
& i \frac{\partial u_{1}}{\partial t}-\varepsilon_{1}(-\Delta)^{\frac{\alpha}{2}} u_{1}+\varpi_{1} u_{1}+\varpi_{2} u_{2}+\varepsilon_{2} f\left(\left|u_{1}\right|^{2},\left|u_{2}\right|^{2}\right) u_{1}=0 \\
& i \frac{\partial u_{2}}{\partial t}-\varepsilon_{3}(-\Delta)^{\frac{\alpha}{2}} u_{2}+\varpi_{2} u_{1}+\varpi_{1} u_{2}+\varepsilon_{4} g\left(\left|u_{1}\right|^{2},\left|u_{2}\right|^{2}\right) u_{2}=0 .
\end{aligned}
$$

To define the DDG method, we rewrite (7.1) as:

$$
\begin{aligned}
& i \frac{\partial u_{1}}{\partial t}+\varepsilon_{1} e+\varpi_{1} u_{1}+\varpi_{2} u_{2}+\varepsilon_{2} f\left(\left|u_{1}\right|^{2},\left|u_{2}\right|^{2}\right) u_{1}=0, \\
& e=\Delta_{(\alpha-2) / 2} r, \quad r=\frac{\partial^{2}}{\partial x^{2}} u_{1}, \\
& i \frac{\partial u_{2}}{\partial t}+\varepsilon_{3} l+\varpi_{2} u_{1}+\varpi_{1} u_{2}+\varepsilon_{4} g\left(\left|u_{1}\right|^{2},\left|u_{2}\right|^{2}\right) u_{2}=0, \\
& l=\Delta_{(\alpha-2) / 2} w, \quad w=\frac{\partial^{2}}{\partial x^{2}} u_{2} .
\end{aligned}
$$


We decompose the complex functions $u(x, t)$ and $v(x, t)$ into their real and imaginary parts. Setting $u_{1}(x, t)=$ $p(x, t)+i q(x, t)$ and $u_{2}(x, t)=v(x, t)+i \theta(x, t)$ in system [7.1), we can obtain the following coupled system

$$
\begin{aligned}
& \frac{\partial p}{\partial t}+\varepsilon_{1} Q+\varpi_{1} q+\varpi_{2} \theta+\varepsilon_{2} f\left(\left|u_{1}\right|^{2},\left|u_{2}\right|^{2}\right) q=0, \\
& Q=\Delta_{(\alpha-2) / 2} r, \quad r=\frac{\partial^{2}}{\partial x^{2}} q, \\
& \frac{\partial q}{\partial t}-\varepsilon_{1} H-\varpi_{1} p-\varpi_{2} v-\varepsilon_{2} f\left(\left|u_{1}\right|^{2},\left|u_{2}\right|^{2}\right) p=0, \\
& H=\Delta_{(\alpha-2) / 2} w, \quad w=\frac{\partial^{2}}{\partial x^{2}} p, \\
& \frac{\partial v}{\partial t}+\varepsilon_{3} L+\varpi_{3} q+\varpi_{4} \theta+\varepsilon_{4} g\left(\left|u_{1}\right|^{2},\left|u_{2}\right|^{2}\right) \theta=0, \\
& L=\Delta_{(\alpha-2) / 2} \rho, \quad \rho=\frac{\partial^{2}}{\partial x^{2}} \theta, \\
& \frac{\partial \theta}{\partial t}-\varepsilon_{3} E-\varpi_{2} p-\varpi_{1} v-\varepsilon_{4} g\left(\left|u_{1}\right|^{2},\left|u_{2}\right|^{2}\right) v=0, \\
& E=\Delta_{(\alpha-2) / 2} \xi, \quad \xi=\frac{\partial^{2}}{\partial x^{2}} v .
\end{aligned}
$$

We now define DDG scheme as follows: find $p_{h}, q_{h}, Q_{h}, r_{h}, H_{h}, w_{h}, v_{h}, \theta_{h}, L_{h}, \rho_{h}, E_{h}, \xi_{h} \in V_{k}^{N}$, such that for all test functions $\vartheta_{1}, \beta_{1}, \phi, \chi, \beta_{2}, \psi, \gamma, \beta_{3}, \delta, o, \beta_{4}, \omega \in V_{k}^{N}$,

$$
\begin{aligned}
& \left(\frac{\partial p_{h}}{\partial t}, \vartheta_{1}\right)_{D^{k}}+\varepsilon_{1}\left(Q_{h}, \vartheta_{1}\right)_{D^{k}}+\varpi_{1}\left(q_{h}, \vartheta_{1}\right)_{D^{k}}+\varpi_{2}\left(\theta_{h}, \vartheta_{1}\right)_{D^{k}}+\varepsilon_{2}\left(f\left(\left|u_{1}\right|^{2},\left|u_{2}\right|^{2}\right) q_{h}, \vartheta_{1}\right)_{D^{k}}=0, \\
& \left(Q_{h}, \beta_{1}\right)_{D^{k}}=\left(\Delta_{(\alpha-2) / 2} r_{h}, \beta_{1}\right)_{D^{k}}, \\
& \left(r_{h}, \phi\right)_{D^{k}}=-\left(\partial_{x} q_{h}, \partial_{x} \phi\right)_{D^{k}}-\left(\left(\partial_{x} q_{h}\right)^{*}[\phi]+\left\{\partial_{x} \phi\right\}\left[q_{h}\right]\right)_{k+\frac{1}{2}}, \\
& \left(\frac{\partial q_{h}}{\partial t}, \chi\right)_{D^{k}}-\varepsilon_{1}\left(H_{h}, \chi\right)_{D^{k}}-\varpi_{1}\left(p_{h}, \chi\right)_{D^{k}}-\varpi_{2}\left(v_{h}, \chi\right)_{D^{k}}-\varepsilon_{2}\left(f\left(\left|u_{1}\right|^{2},\left|u_{2}\right|^{2}\right) p_{h}, \chi\right)_{D^{k}}=0, \\
& \left(H_{h}, \beta_{2}\right)_{D^{k}}=\left(\Delta_{(\alpha-2) / 2} w_{h}, \beta_{2}\right)_{D^{k}}, \\
& \left(w_{h}, \psi\right)_{D^{k}}=-\left(\partial_{x} p_{h}, \partial_{x} \psi\right)_{D^{k}}-\left(\left(\partial_{x} p_{h}\right)^{*}[\psi]+\left\{\partial_{x} \psi\right\}\left[p_{h}\right]\right)_{k+\frac{1}{2}}, \\
& \left(\frac{\partial v_{h}}{\partial t}, \gamma\right)_{D^{k}}+\varepsilon_{3}\left(L_{h}, \gamma\right)_{D^{k}}+\varpi_{2}\left(q_{h}, \gamma\right)_{D^{k}}+\varpi_{1}\left(\theta_{h}, \gamma\right)_{D^{k}}+\varepsilon_{4}\left(g\left(\left|u_{1}\right|^{2},\left|u_{2}\right|^{2}\right) \theta_{h}, \gamma\right)_{D^{k}}=0, \\
& \left(L_{h}, \beta_{3}\right)_{D^{k}}=\left(\Delta_{(\alpha-2) / 2} \rho_{h}, \beta_{3}\right)_{D^{k}}, \\
& \left(\rho_{h}, \delta\right)_{D^{k}}=-\left(\partial_{x} \theta_{h}, \partial_{x} \delta\right)_{D^{k}}-\left(\left(\partial_{x} \theta_{h}\right)^{*}[\delta]+\left\{\partial_{x} \delta\right\}\left[\theta_{h}\right]\right)_{k+\frac{1}{2}}, \\
& \left(\frac{\partial \theta_{h}}{\partial t}, o\right)_{D^{k}}-\lambda_{3}\left(E_{h}, o\right)_{D^{k}}-\varpi_{2}\left(p_{h}, o\right)_{D^{k}}-\varpi_{1}\left(v_{h}, o\right)_{D^{k}}-\lambda_{4}\left(g\left(\left|u_{1}\right|^{2},\left|u_{2}\right|^{2}\right) v_{h}, o\right)_{D^{k}}=0, \\
& \left(E_{h}, \beta_{4}\right)_{D^{k}}=\left(\Delta_{(\alpha-2) / 2} \xi_{h}, \beta_{4}\right)_{D^{k}}, \\
& \left(\xi_{h}, \omega\right)_{D^{k}}=-\left(\partial_{x} v_{h}, \partial_{x} \omega\right)_{D^{k}}-\left(\left(\partial_{x} v_{h}\right)^{*}[\omega]+\left\{\partial_{x} \omega\right\}\left[v_{h}\right]\right)_{k+\frac{1}{2}}
\end{aligned}
$$


Theorem 7.1. ( $L^{2}$ stability). Suppose $u_{1}(x, t)=p(x, t)+i q(x, t)$ and $u_{2}(x, t)=v(x, t)+i \theta(x, t)$ and let $u_{1 h}, u_{2 h} \in V_{k}^{N}$ be the approximation of $u_{1}, u_{2}$ then the solution to the scheme (7.4) satisfies the $L^{2}$ stability

$$
\left\|u_{1 h}\right\|_{L^{2}(\Omega)}^{2}+\left\|u_{2 h}\right\|_{L^{2}(\Omega)}^{2} \leq C\left(\left\|u_{1 h}(x, 0)\right\|_{L^{2}(\Omega)}^{2}+\left\|u_{2 h}(x, 0)\right\|_{L^{2}(\Omega)}^{2}\right) .
$$

Theorem 7.2. Let $u_{1}$ and $u_{2}$ be the exact solutions of the linear coupled fractional Schrödinger equations (7.1), and let $u_{1 h}$ and $u_{2 h}$ be the numerical solutions of the DDG scheme 7.4. Then for small enough $h$, we have the following error estimates:

$$
\left\|u_{1}(., T)-u_{1 h}(., T)\right\|_{L^{2}(\Omega)}+\left\|u_{2}(., T)-u_{2 h}(., T)\right\|_{L^{2}(\Omega)} \leq C h^{N+1} .
$$

Theorem 7.2 and 7.1 can be proven by similar techniques as that in the proof of Theorem 6.1 and 6.2 . We will thus not give the details here.

\section{Numerical examples}

In the following, we present some numerical experiments to show the accuracy and the performance of the present DDG method for the fractional convection-diffusion and Schrödinger type equations. To deal with the method-of-line fractional PDE, i.e., the classical ODE system, we use the high-order Runge-Kutta time discretizations [59], when the polynomials are of degree $N$, a higher-order accurate Runge-Kutta (RK) method must be used in order to guarantee that the scheme is stable. In this paper we use a fourth-order non-Total variation diminishing (TVD) Runge-Kutta scheme [60. Numerical experiments demonstrate its numerical stability

$$
\frac{\partial \mathbf{u}_{h}}{\partial t}=\mathcal{F}\left(\mathbf{u}_{h}, t\right)
$$

where $\mathbf{u}_{h}$ is the vector of unknowns, we can use the standard fourth-order four stage explicit RK method (ERK)

$$
\begin{aligned}
& \mathbf{k}^{1}=\mathcal{F}\left(\mathbf{u}_{h}^{n}, t^{n}\right), \\
& \mathbf{k}^{2}=\mathcal{F}\left(\mathbf{u}_{h}^{n}+\frac{1}{2} \Delta t \mathbf{k}^{1}, t^{n}+\frac{1}{2} \Delta t\right), \\
& \mathbf{k}^{3}=\mathcal{F}\left(\mathbf{u}_{h}^{n}+\frac{1}{2} \Delta t \mathbf{k}^{2}, t^{n}+\frac{1}{2} \Delta t\right), \\
& \mathbf{k}^{4}=\mathcal{F}\left(\mathbf{u}_{h}^{n}+\Delta t \mathbf{k}^{3}, t^{n}+\Delta t\right), \\
& \mathbf{u}_{h}^{n+1}=\mathbf{u}_{h}^{n}+\frac{1}{6}\left(\mathbf{k}^{1}+2 \mathbf{k}^{2}+2 \mathbf{k}^{3}+\mathbf{k}^{4}\right),
\end{aligned}
$$

to advance from $\mathbf{u}_{h}^{n}$ to $\mathbf{u}_{h}^{n+1}$, separated by the time step, $\Delta t$. In our examples, the condition $\Delta t \leq C \Delta x_{\min }^{\alpha}(0<$ $C<1)$ is used to ensure stability.

Example 8.1. Consider the fractional diffusion equation

$$
\begin{aligned}
& \frac{\partial u(x, t)}{\partial t}+\varepsilon(-\Delta)^{\frac{\alpha}{2}} u(x, t)=g(x, t), \quad x \in[-1,1], \quad t \in(0,0.5], \\
& u(x, 0)=u_{0}(x)
\end{aligned}
$$


with the initial condition $u_{0}(x)=\left(x^{2}-1\right)^{4}$ and the corresponding forcing term $g(x, t)$ is of the form

$$
g(x, t)=e^{-t}\left(-u_{0}(x)+\varepsilon(-\Delta)^{\frac{\alpha}{2}} u_{0}(x)\right)
$$

then the exact solution is $u(x, t)=e^{-t}\left(x^{2}-1\right)^{4}$ with $\varepsilon=\frac{\Gamma(9-\alpha)}{\Gamma(9)}$.

We solve the equation for several different $\alpha$ and polynomial orders. Moreover, we use numerical flux (3.9) with $\beta_{1}=0$ and $\beta_{0}$ is a parameter depending on the degree of the approximation polynomial in [52]. The errors and order of convergence are listed in Table 1 and show that the DDG method can achieve the accuracy of order $N+1$.

Example 8.2. we consider the fractional diffusion equation

$$
\begin{aligned}
& \frac{\partial u(x, t)}{\partial t}+\varepsilon(-\Delta)^{\frac{\alpha}{2}} u(x, t)=g(x, t), \quad x \in[0,1], \quad t \in(0,0.5], \\
& u(x, 0)=u_{0}(x)
\end{aligned}
$$

with the initial condition $u_{0}(x)=x^{11}$ and the Dirichlet boundary conditions $u(0, t)=0, u(1, t)=e^{-t}$. The corresponding forcing term $g(x, t)$ is of the form

$$
g(x, t)=e^{-t}\left(-u_{0}(x)+(-\Delta)^{\frac{\alpha}{2}} u_{0}(x)\right),
$$

then the exact solution is $u(x, t)=e^{-t} x^{11}$ with $\alpha=1.1, \varepsilon=\frac{\Gamma(12-\alpha)}{\Gamma(12)}$. We consider cases with $N=3,4,5$, $K=10,15,20,25$. The numerical orders of convergence are shown in Figure 1, showing an $O\left(h^{N+1}\right)$ convergence rate for all orders.

Example 8.3. We consider the fractional Burgers' equation

$$
\begin{aligned}
& \frac{\partial u(x, t)}{\partial t}+\varepsilon(-\Delta)^{\frac{\alpha}{2}} u(x, t)+\frac{\partial}{\partial x}\left(\frac{u^{2}(x, t)}{2}\right)=g(x, t), \quad x \in[-1,1], \quad t \in(0,1], \\
& u(x, 0)=u_{0}(x)
\end{aligned}
$$

with the initial condition $u_{0}(x)=\frac{\left(x^{2}-1\right)^{4}}{100}$ and the corresponding forcing term $g(x, t)$ is of the form

$$
g(x, t)=e^{-t}\left(-u_{0}(x)+e^{-t} u_{0}(x) u_{0}^{\prime}(x) u_{0}(x)+\varepsilon(-\Delta)^{\frac{\alpha}{2}} u_{0}(x)\right) .
$$

In this case, the exact solution will be $u(x, t)=\frac{e^{-t}\left(x^{2}-1\right)^{4}}{100}$.

To complete the scheme, we choose a Lax-Friedrichs flux for the nonlinear term. The problem is solved for several different values of $\alpha$, polynomial orders $(N)$, and numbers of elements $(K)$. Table 2 shows the numerical $L^{2}$-Error and the convergence rates of the DDG method with numerical flux $(3.9)$ when $\beta_{0}=1$ and $\beta_{1}=\frac{1}{12}$. From there we see that the DDG method can achieve the accuracy of order $N+1$. 


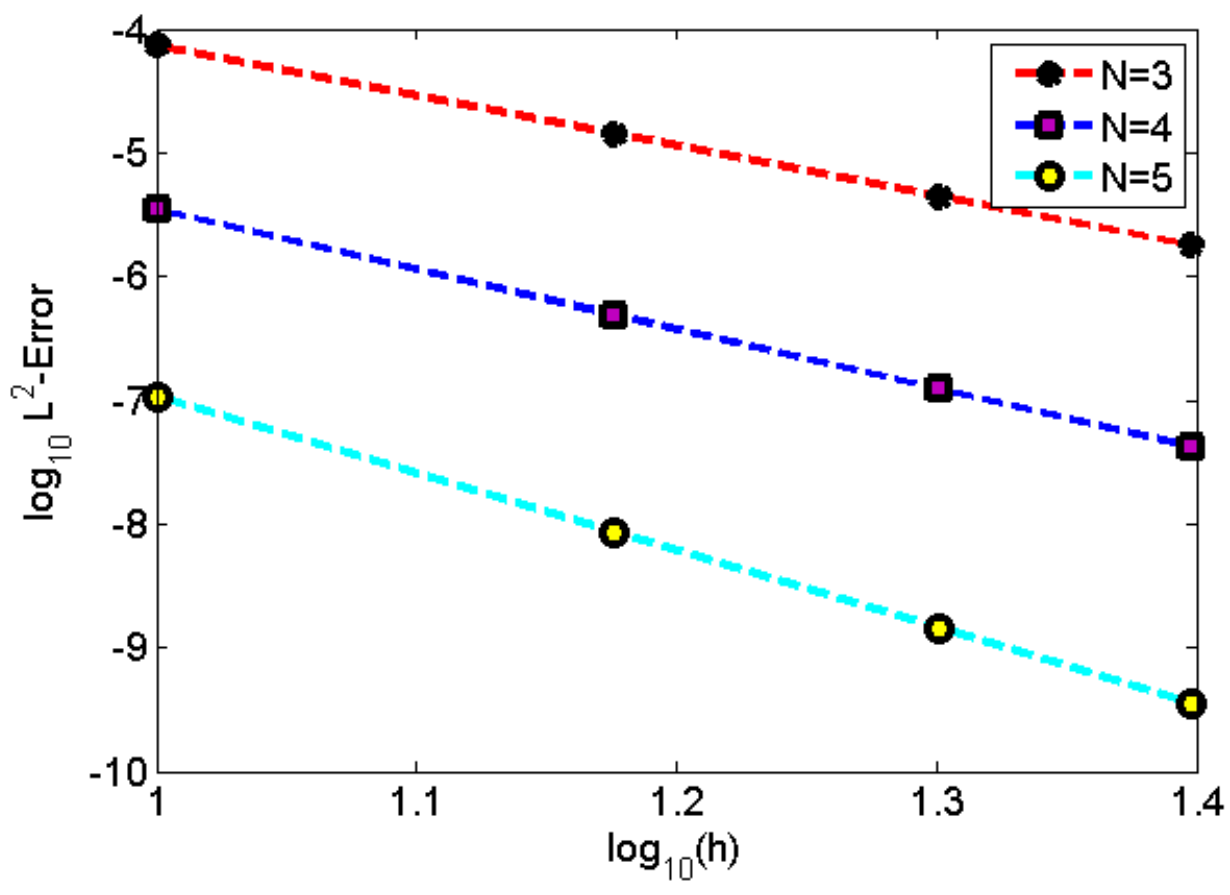

Figure 1: Convergence tests of 8.2 with different values of $N$ and $K$. 


\begin{tabular}{|c|c|c|c|c|c|c|c|}
\hline \multirow{3}{*}{$\begin{array}{l}\mathrm{K} \\
\alpha\end{array}$} & \multicolumn{7}{|c|}{$N=1$} \\
\hline & $2^{4}$ & \multicolumn{2}{|c|}{$2^{5}$} & \multicolumn{2}{|c|}{$2^{6}$} & \multicolumn{2}{|c|}{$2^{7}$} \\
\hline & $L^{2}$-Error & $L^{2}$-Error & order & $L^{2}$-Error & order & $L^{2}$-Error & order \\
\hline 1.1 & $4.5 \mathrm{e}-03$ & $7.65 \mathrm{e}-04$ & 2.55 & $2.16 \mathrm{e}-04$ & 1.82 & $5.07 \mathrm{e}-05$ & 2.09 \\
\hline 1.3 & $5.0 \mathrm{e}-03$ & $1.3 \mathrm{e}-03$ & 1.98 & $3.42 \mathrm{e}-04$ & 1.88 & $8.36 \mathrm{e}-05$ & 2.03 \\
\hline \multirow[t]{2}{*}{1.6} & 8.4 e- 03 & $2.7 \mathrm{e}-03$ & 1.66 & $7.64 \mathrm{e}-04$ & 1.78 & $2.11 \mathrm{e}-04$ & 1.86 \\
\hline & \multicolumn{7}{|c|}{$N=2$} \\
\hline $\mathrm{K}$ & $2^{4}$ & \multicolumn{2}{|c|}{$2^{5}$} & \multicolumn{2}{|c|}{$2^{6}$} & \multicolumn{2}{|c|}{$2^{7}$} \\
\hline$\alpha$ & $L^{2}$-Error & $L^{2}$-Error & order & $L^{2}$-Error & order & $L^{2}$-Error & order \\
\hline 1.1 & $3.3 \mathrm{e}-03$ & $2.89 \mathrm{e}-04$ & 3.51 & $2.73 \mathrm{e}-05$ & 3.41 & $2.60 \mathrm{e}-06$ & 3.39 \\
\hline 1.3 & $2.5 \mathrm{e}-03$ & $2.03 \mathrm{e}-04$ & 3.65 & $1.86 \mathrm{e}-05$ & 3.44 & $1.79 \mathrm{e}-06$ & 3.38 \\
\hline \multirow[t]{2}{*}{1.6} & $1.2 \mathrm{e}-03$ & $1.04 \mathrm{e}-04$ & 3.58 & $9.21 \mathrm{e}-06$ & 3.49 & $8.61 \mathrm{e}-07$ & 3.42 \\
\hline & \multicolumn{7}{|c|}{$N=3$} \\
\hline $\mathrm{K}$ & $2^{4}$ & \multicolumn{2}{|c|}{$2^{5}$} & \multicolumn{2}{|c|}{$2^{6}$} & \multicolumn{2}{|c|}{$2^{7}$} \\
\hline$\alpha$ & $L^{2}$-Error & $L^{2}$-Error & order & $L^{2}$-Error & order & $L^{2}$-Error & order \\
\hline 1.1 & $1.84 \mathrm{e}-05$ & $1.12 \mathrm{e}-06$ & 4.05 & $5.55 \mathrm{e}-08$ & 4.32 & $2.59 \mathrm{e}-09$ & 4.42 \\
\hline 1.3 & $2.03 \mathrm{e}-05$ & $1.22 \mathrm{e}-06$ & 4.06 & $6.46 \mathrm{e}-08$ & 4.24 & $3.96 \mathrm{e}-09$ & 4.03 \\
\hline 1.6 & $2.35 \mathrm{e}-05$ & $1.45 \mathrm{e}-06$ & 4.02 & $9.38 \mathrm{e}-08$ & 3.95 & $6.22 \mathrm{e}-09$ & 3.92 \\
\hline & \multicolumn{7}{|c|}{$N=4$} \\
\hline K & 20 & \multicolumn{2}{|c|}{25} & \multicolumn{2}{|c|}{30} & \multicolumn{2}{|c|}{35} \\
\hline$\alpha$ & $L^{2}$-Error & $L^{2}$-Error & order & $L^{2}$-Error & order & $L^{2}$-Error & order \\
\hline 1.1 & $3.72 \mathrm{e}-07$ & $1.27 \mathrm{e}-07$ & 4.82 & $5.23 \mathrm{e}-08$ & 4.86 & $2.47 \mathrm{e}-08$ & 4.86 \\
\hline 1.3 & $3.98 \mathrm{e}-07$ & $1.35 \mathrm{e}-07$ & 4.84 & $5.54 \mathrm{e}-08$ & 4.88 & $2.60 \mathrm{e}-08$ & 4.91 \\
\hline 1.6 & $4.34 \mathrm{e}-07$ & $1.47 \mathrm{e}-07$ & 4.85 & $6.04 \mathrm{e}-08$ & 4.88 & $2.84 \mathrm{e}-08$ & 4.9 \\
\hline
\end{tabular}

Table 1: Numerical results for the fractional diffusion equation in Example 8.1

Example 8.4. We consider the fractional Burgers' equation

$$
\begin{aligned}
& \frac{\partial u(x, t)}{\partial t}+\varepsilon(-\Delta)^{\frac{\alpha}{2}} u(x, t)+\frac{\partial}{\partial x}\left(\frac{u^{2}(x, t)}{2}\right)=g(x, t), \quad x \in[0,1], \quad t \in(0,1], \\
& u(x, 0)=u_{0}(x),
\end{aligned}
$$

with the initial condition $u_{0}(x)=\frac{x^{4}}{100}$ and the corresponding forcing term $g(x, t)$ is of the form

$$
g(x, t)=e^{-t}\left(-u_{0}(x)+e^{-t} u_{0}(x) u_{0}^{\prime}(x)+\varepsilon(-\Delta)^{\frac{\alpha}{2}} u_{0}(x)\right) .
$$




\begin{tabular}{|c|c|c|c|c|c|c|c|}
\hline & \multicolumn{7}{|c|}{$N=1$} \\
\hline \multirow{2}{*}{$\begin{array}{l}\mathrm{K} \\
\alpha\end{array}$} & 10 & \multicolumn{2}{|c|}{20} & \multicolumn{2}{|c|}{30} & \multicolumn{2}{|c|}{40} \\
\hline & $L^{2}$-Error & $L^{2}$-Error & order & $L^{2}$-Error & order & $L^{2}$-Error & order \\
\hline 1.1 & $2.76 \mathrm{e}-04$ & $3.15 \mathrm{e}-05$ & 3.13 & $8.97 \mathrm{e}-06$ & 3.1 & $4.68 \mathrm{e}-06$ & 2.26 \\
\hline 1.3 & $1.43 \mathrm{e}-04$ & $3.05 \mathrm{e}-05$ & 2.23 & $1.34 \mathrm{e}-05$ & 2.03 & $7.88 \mathrm{e}-06$ & 1.85 \\
\hline \multirow[t]{2}{*}{1.6} & $1.51 \mathrm{e}-04$ & $5.75 \mathrm{e}-05$ & 1.5 & $2.92 \mathrm{e}-05$ & 1.7 & $1.7 \mathrm{e}-05$ & 1.9 \\
\hline & \multicolumn{7}{|c|}{$N=2$} \\
\hline $\mathrm{K}$ & 10 & \multicolumn{2}{|c|}{20} & \multicolumn{2}{|c|}{30} & \multicolumn{2}{|c|}{40} \\
\hline$\alpha$ & $L^{2}$-Error & $L^{2}$-Error & order & $L^{2}$-Error & order & $L^{2}$-Error & order \\
\hline 1.1 & $1.35 \mathrm{e}-04$ & $1.82 \mathrm{e}-05$ & 2.89 & $5.45 \mathrm{e}-06$ & 2.9717 & $2.32 \mathrm{e}-06$ & 2.98 \\
\hline 1.3 & $8.1 \mathrm{e}-05$ & $1.16 \mathrm{e}-05$ & 2.78 & $3.57 \mathrm{e}-06$ & 2.91 & $1.54 \mathrm{e}-06$ & 2.92 \\
\hline 1.6 & $2.88 \mathrm{e}-05$ & $4.43 \mathrm{e}-06$ & 2.70 & $1.39 \mathrm{e}-06$ & 2.89 & $6.07 \mathrm{e}-07$ & 2.88 \\
\hline
\end{tabular}

Table 2: $\quad L^{2}$-Error and order of convergence for Example 8.3 with $K$ elements and polynomial order $N$.

In this case, the exact solution will be $u(x, t)=\frac{e^{-t} x^{4}}{100}$ and $\varepsilon=\frac{\Gamma(5-\alpha)}{\Gamma(5)}$. Table 3 shows the numerical $L^{2}$ -Error and the convergence rates of the DDG method. From there we see that the DDG method can achieve the accuracy of order $N+1$.

Example 8.5. We consider the fractional convection-diffusion equation with a discontinuous initial condition,

$$
u(x, 0)= \begin{cases}x+1, & -1 \leq x<0 \\ 2 x, & 0 \leq x \leq 1 \\ 0, & \text { otherwise }\end{cases}
$$

We consider 1.1] with parameters $\varepsilon=1, x \in[-10,10]$ and solve the equation for several different values of $\alpha$. The numerical solution $u_{h}(x, t)$ for $\alpha=1.2,1.4,1.6,1.8,2.0$ is shown in figure 2. From this figure clear that the dissipative effect increases with $\alpha$ and the classical case with $\alpha=2$ is a limit of the fractional case.

Example 8.6. We consider the fractional convection-diffusion equation (1.1) with initial condition,

$$
u(x, 0)=e^{-2 x^{2}},
$$

with parameters $\varepsilon=1, x \in[-10,10]$. We consider cases with $N=2$ and $K=50$ and solve the equation for several different values of $\alpha$. The numerical solution $u_{h}(x, t)$ for $\alpha=1.2,1.4,1.6,1.8,2.0$ is shown in Figure 3 . We observe that the order $\alpha$ will affect the shape of the soliton case. This property of the fractional convectiondiffusion equation can be used in physics to modify the shape of wave without change of the nonlinearity and dispersion effects. The numerical solutions of the fractional equation are convergent to the solutions of the classical non-fractional equation when $\alpha$ tends to 2 . 


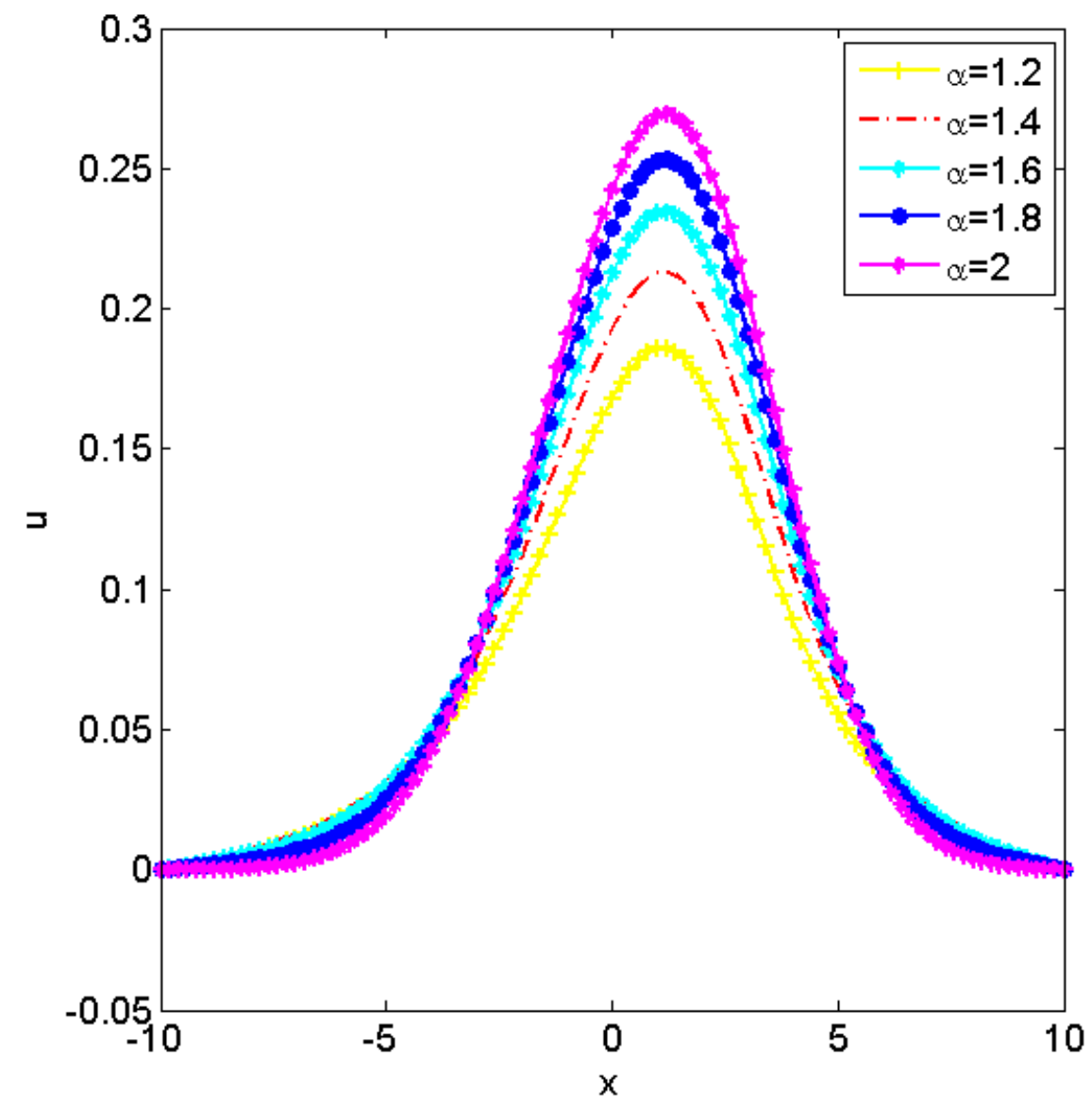

Figure 2: The profile of $u$ with different fractional order $\alpha$ for smooth initial function at $T=3$. 


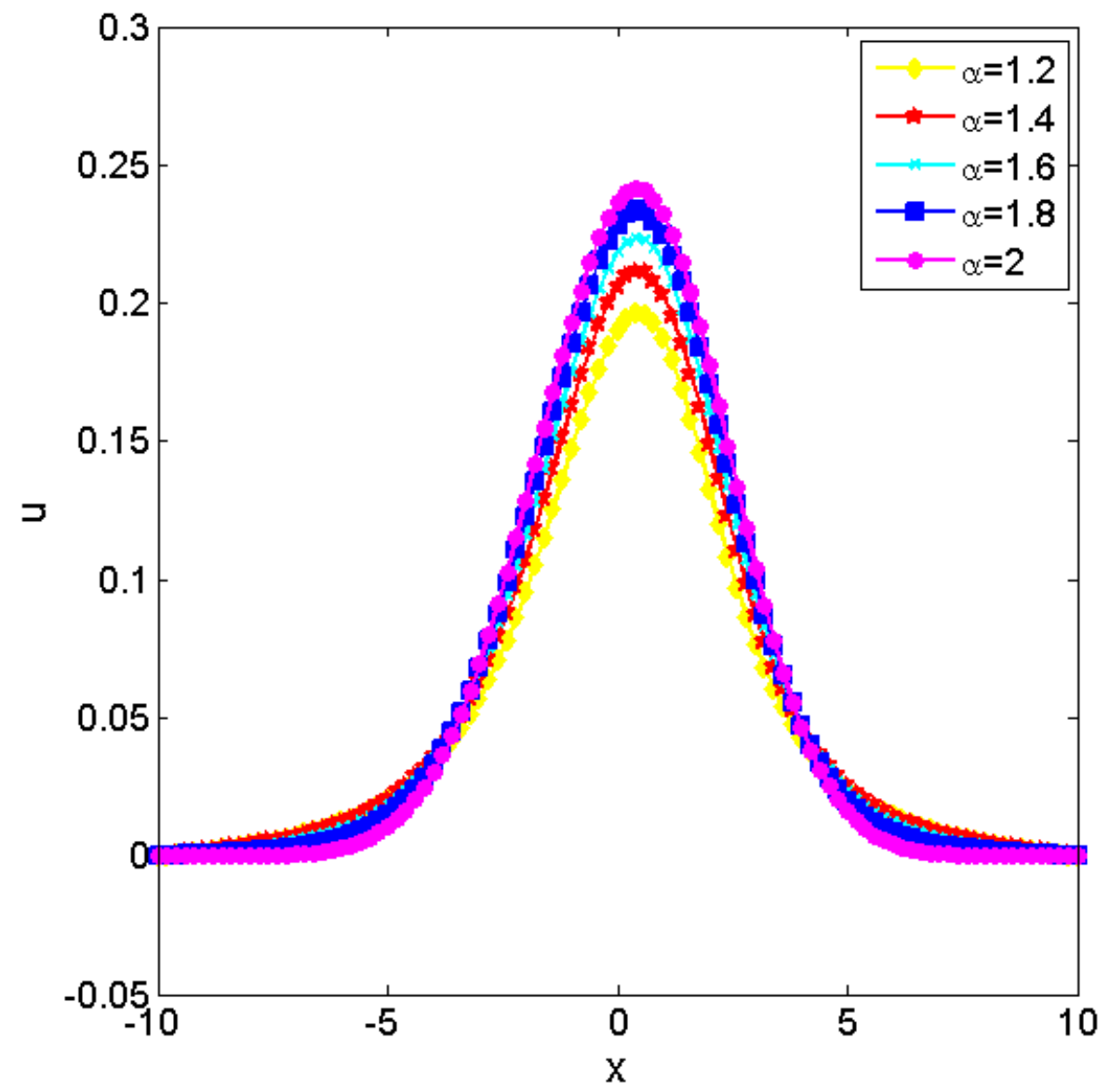

Figure 3: The profile of $u$ with different fractional order $\alpha$ for smooth initial function at $T=3$. 


\begin{tabular}{|c|c|c|c|c|c|c|c|}
\hline \multirow{3}{*}{$\begin{array}{l}\mathrm{K} \\
\alpha\end{array}$} & \multicolumn{7}{|c|}{$N=2$} \\
\hline & 10 & \multicolumn{2}{|c|}{20} & \multicolumn{2}{|c|}{30} & \multicolumn{2}{|c|}{40} \\
\hline & $L^{2}$-Error & $L^{2}$-Error & order & $L^{2}$-Error & order & $L^{2}$-Error & order \\
\hline 1.2 & $1.11 \mathrm{e}-05$ & $1.54 \mathrm{e}-06$ & 2.85 & $4.54 \mathrm{e}-07$ & 3.01 & $1.87 \mathrm{e}-07$ & 3.09 \\
\hline 1.4 & $7.03 \mathrm{e}-05$ & $1.02 \mathrm{e}-05$ & 2.79 & $3.24 \mathrm{e}-06$ & 2.83 & $1.4 \mathrm{e}-06$ & 2.9 \\
\hline 1.6 & $6.55 \mathrm{e}-05$ & $9.77 \mathrm{e}-06$ & 2.74 & $3.13 \mathrm{e}-06$ & 2.81 & $1.39 \mathrm{e}-06$ & 2.82 \\
\hline \multirow[t]{2}{*}{1.8} & $6.21 \mathrm{e}-05$ & $9.39 \mathrm{e}-06$ & 2.73 & $3.03 \mathrm{e}-06$ & 2.79 & $1.37 \mathrm{e}-06$ & 2.75 \\
\hline & \multicolumn{7}{|c|}{$N=3$} \\
\hline K & 20 & \multicolumn{2}{|c|}{25} & \multicolumn{2}{|r|}{30} & \multicolumn{2}{|c|}{35} \\
\hline$\alpha$ & $L^{2}$-Error & $L^{2}$-Error & order & $L^{2}$-Error & order & $L^{2}$-Error & order \\
\hline 1.2 & $2.46 \mathrm{e}-06$ & $2.07 \mathrm{e}-07$ & 3.57 & $3.92 \mathrm{e}-08$ & 4.1 & $1.168 \mathrm{e}-08$ & 4.21 \\
\hline 1.4 & $2.98 \mathrm{e}-06$ & $2.33 \mathrm{e}-07$ & 3.67 & $4.64 \mathrm{e}-08$ & 3.98 & $1.43 \mathrm{e}-08$ & 4.09 \\
\hline 1.6 & $2.9 \mathrm{e}-06$ & $2.28 \mathrm{e}-07$ & 3.67 & $4.61 \mathrm{e}-08$ & 2.81 & $1.48 \mathrm{e}-08$ & 3.91 \\
\hline 1.8 & $2.71 \mathrm{e}-06$ & $2.06 \mathrm{e}-07$ & 3.72 & $4.32 \mathrm{e}-08$ & 3.85 & $1.38 \mathrm{e}-08$ & 3.98 \\
\hline
\end{tabular}

Table 3: $\quad L^{2}$-Error and order of convergence for Example 8.4 with $K$ elements and polynomial order $N$.

Example 8.7. We consider the nonlinear fractional Schrödinger equation

$$
\begin{aligned}
& i \frac{\partial u}{\partial t}-\varepsilon(-\Delta)^{\frac{\alpha}{2}} u+|u|^{2} u=g(x, t), \quad x \in[-1,1], \quad t \in(0,0.5], \\
& u(x, 0)=u_{0}(x),
\end{aligned}
$$

with the initial condition $u_{0}(x)=\left(x^{2}-1\right)^{5}$ and the corresponding forcing term $g(x, t)$ is of the form

$$
g(x, t)=e^{-i t}\left(i u_{0}(x)-\varepsilon(-\Delta)^{\frac{\alpha}{2}} u_{0}(x)+\left(u_{0}(x)\right)^{3}\right)
$$

to obtain an exact solution $u(x, t)=e^{-i t}\left(x^{2}-1\right)^{5}$ with $\varepsilon=\frac{\Gamma(11-\alpha)}{\Gamma(11)}$. We solve the equation for several dierent and polynomial orders. The errors and order of convergence are listed in Table 4 confirming optimal $O\left(h^{N+1}\right)$ order of convergence across $1<\alpha<2$.

Example 8.8. We consider the nonlinear fractional Schrödinger equation (5.1) with initial conditions:

(a) Single soliton: We consider the initial condition

$$
u(x, 0)=e^{2 i\left(x-x_{0}\right)} \operatorname{sech}\left(x-x_{0}\right),
$$

with parameters $\varepsilon_{1}=\varepsilon_{2}=2, x_{0}=0$ and $x \in[-25,25]$. We consider cases with $N=2$ and $K=200$ and solve the equation for several different values of $\alpha$. The numerical solution $u_{h}(x, t)$ for $\alpha=1.4,1.6,1.8,2.0$ is shown in Figure 4. We observe that the order $\alpha$ will affect the shape of the soliton case. When $\alpha$ becomes smaller, the 


\begin{tabular}{|c|c|c|c|c|c|c|c|}
\hline \multirow{3}{*}{$\begin{array}{l}\mathrm{K} \\
\alpha\end{array}$} & \multicolumn{7}{|c|}{$N=1$} \\
\hline & $2^{4}$ & \multicolumn{2}{|c|}{$2^{5}$} & \multicolumn{2}{|c|}{$2^{6}$} & \multicolumn{2}{|c|}{$2^{7}$} \\
\hline & $L^{2}$-Error & $L^{2}$-Error & order & $L^{2}$-Error & order & $L^{2}$-Error & order \\
\hline 1.1 & $1.6 \mathrm{e}-03$ & $3.49 \mathrm{e}-04$ & 2.19 & $6.92 \mathrm{e}-05$ & 2.33 & $1.34 \mathrm{e}-05$ & 2.37 \\
\hline 1.3 & $6.2 \mathrm{e}-03$ & $1.6 \mathrm{e}-03$ & 1.94 & $3.73 \mathrm{e}-04$ & 2.12 & $8.29 \mathrm{e}-05$ & 2.17 \\
\hline \multirow[t]{2}{*}{1.6} & $9.0 \mathrm{e}-03$ & $2.9 \mathrm{e}-03$ & 1.65 & $8.12 \mathrm{e}-04$ & 1.83 & $1.94 \mathrm{e}-04$ & 2.07 \\
\hline & \multicolumn{7}{|c|}{$N=2$} \\
\hline $\mathrm{K}$ & $2^{4}$ & \multicolumn{2}{|c|}{$2^{5}$} & \multicolumn{2}{|c|}{$2^{6}$} & \multicolumn{2}{|c|}{$2^{7}$} \\
\hline$\alpha$ & $L^{2}$-Error & $L^{2}$-Error & order & $L^{2}$-Error & order & $L^{2}$-Error & order \\
\hline 1.1 & $5.52 \mathrm{e}-04$ & $5.75 \mathrm{e}-05$ & 3.27 & $1.03 \mathrm{e}-05$ & 2.48 & $5.44 \mathrm{e}-07$ & 4.25 \\
\hline 1.3 & $6.23 \mathrm{e}-04$ & $7.68 \mathrm{e}-05$ & 3.02 & $1.04 \mathrm{e}-05$ & 2.88 & $1.31 \mathrm{e}-06$ & 3.0 \\
\hline \multirow[t]{2}{*}{1.6} & $6.0 \mathrm{e}-04$ & $7.82 \mathrm{e}-05$ & 2.94 & $9.67 \mathrm{e}-06$ & 3.02 & $1.18 \mathrm{e}-06$ & 3.04 \\
\hline & \multicolumn{7}{|c|}{$N=3$} \\
\hline K & $2^{4}$ & \multicolumn{2}{|c|}{$2^{5}$} & \multicolumn{2}{|c|}{$2^{6}$} & \multicolumn{2}{|c|}{$2^{7}$} \\
\hline$\alpha$ & $L^{2}$-Error & $L^{2}$-Error & order & $L^{2}$-Error & order & $L^{2}$-Error & order \\
\hline 1.1 & $2.47 \mathrm{e}-05$ & $1.62 \mathrm{e}-06$ & 3.93 & $8.86 \mathrm{e}-08$ & 4.19 & $5.52 \mathrm{e}-09$ & 4.0 \\
\hline 1.3 & $2.50 \mathrm{e}-05$ & $1.62 \mathrm{e}-06$ & 3.95 & $1.016 \mathrm{e}-07$ & 4.0 & $6.142 \mathrm{e}-09$ & 4.05 \\
\hline 1.6 & $2.5 \mathrm{e}-05$ & $1.51 \mathrm{e}-06$ & 4.06 & $9.52 \mathrm{e}-08$ & 3.98 & $5.43 \mathrm{e}-09$ & 4.13 \\
\hline
\end{tabular}

Table 4: $\quad$ Numerical results for the nonlinear fractional Schrödinger equation in Example 8.7

shape of the soliton will change more quickly. This property of the fractional Schrödinger equation can be used in physics to modify the shape of wave without change of the nonlinearity and dispersion effects. The numerical solutions of the fractional equation are convergent to the solutions of the classical non-fractional equation when $\alpha$ tends to 2 . 

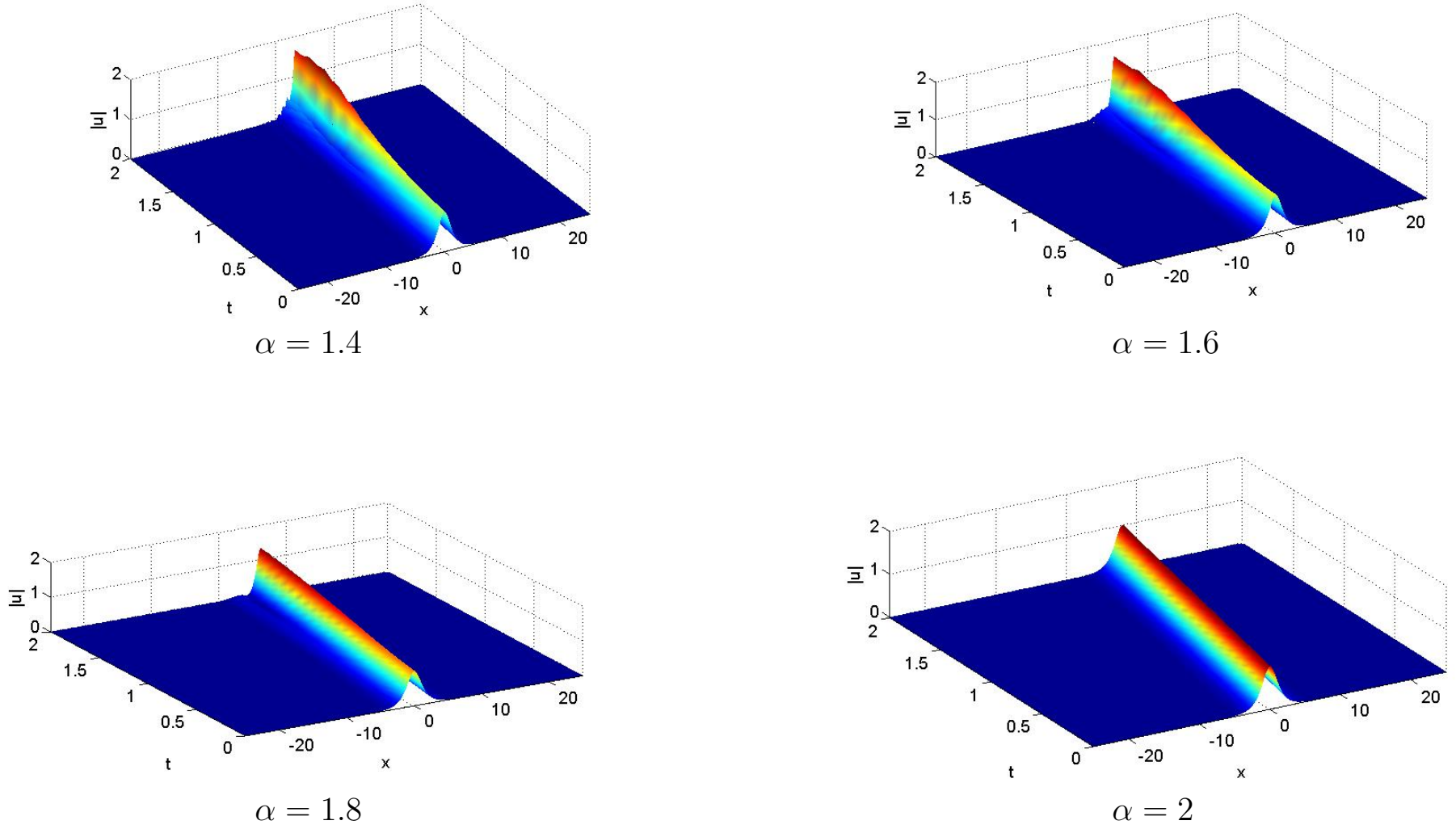

Figure 4: Numerical results for the nonlinear fractional Schrödinger equation in Example 8.8 

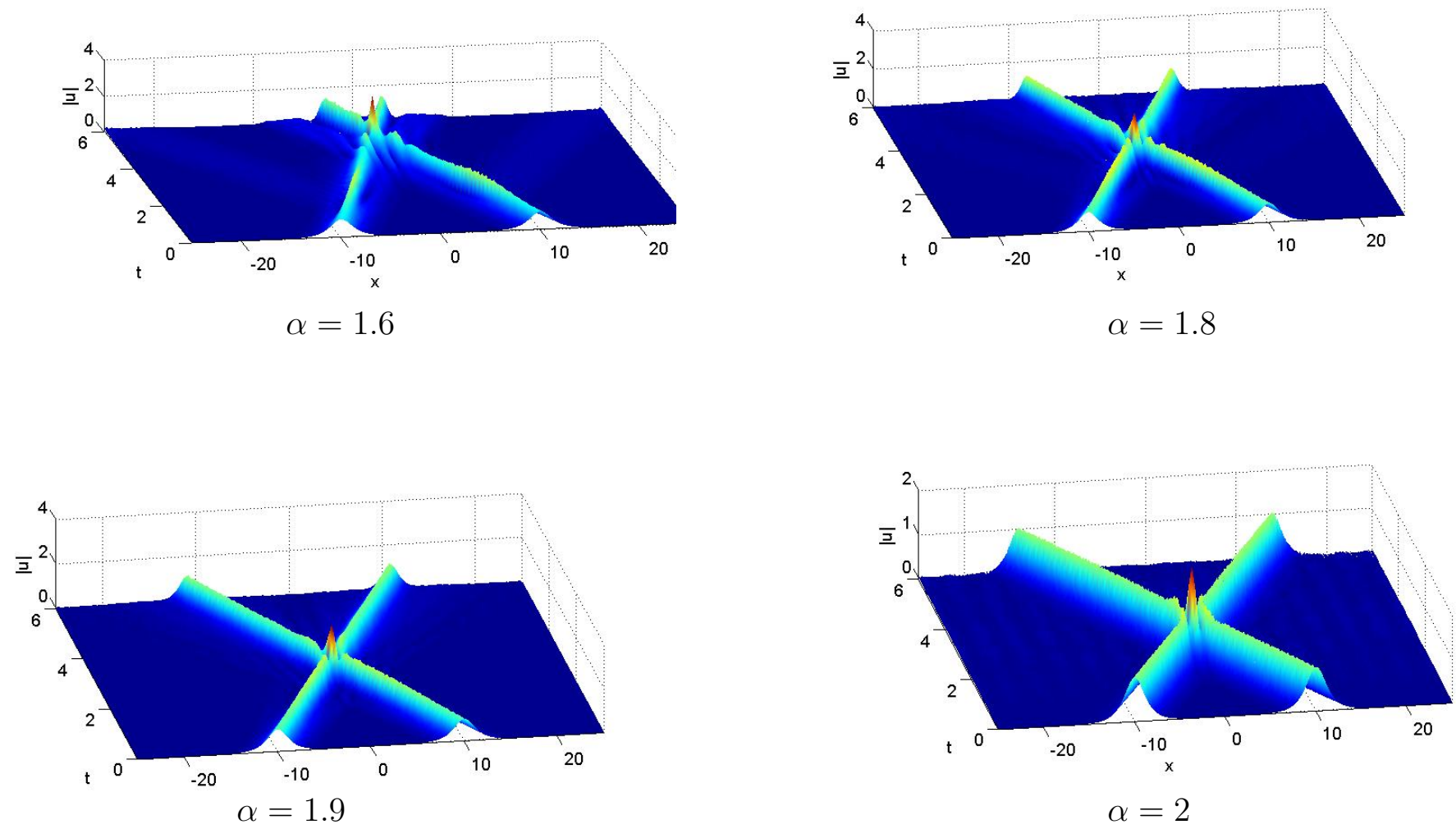

Figure 5: The double soliton collision of 5.1 with initial condition $8.15, c_{1}=4, x_{1}=-10, c_{2}=-4, x_{2}=10$.

(b) Interaction of two solitons: To study the interaction of two solitons, we will take equation (5.1) with the initial condition

$$
u(x, 0)=\sum_{j=1}^{2} e^{\frac{1}{2} i c_{j}\left(x-x_{j}\right)} \operatorname{sech}\left(x-x_{j}\right),
$$

with parameters $\varepsilon_{1}=1, \varepsilon_{2}=2$ and $x \in[-25,25]$. We consider cases with $N=2$ and $K=200$ and solve the equation for several different values of $\alpha$. The numerical solution $u_{h}(x, t)$ for $\alpha=1.6,1.8,1.9,2.0$ is shown in Figure 5. We observe that the order $\alpha$ will affect the shape of the two solitons case and the two waves approach each other interact and leave the interaction unchanged in the shape and velocity. In addition, the interaction is strictly elastic because each of them recovers its exact initial shape after they pass through each other.

Example 8.9. We consider the nonlinear coupled fractional Schrödinger equations

$$
\begin{aligned}
& i \frac{\partial u_{1}(x, t)}{\partial t}-\varepsilon_{1}(-\Delta)^{\frac{\alpha}{2}} u_{1}(x, t)+u_{2}(x, t)+u_{1}(x, t)+\left(\left|u_{1}(x, t)\right|^{2}+\left|u_{2}(x, t)\right|^{2}\right) u_{1}(x, t)=g_{1}(x, t), x \in[0,1], t \in(0,0.5], \\
& i \frac{\partial u_{2}(x, t)}{\partial t}-\varepsilon_{2}(-\Delta)^{\frac{\alpha}{2}} u_{2}(x, t)+u_{2}(x, t)+u_{1}(x, t)+\left(\left|u_{1}(x, t)\right|^{2}+\left|u_{2}(x, t)\right|^{2}\right) u_{2}(x, t)=g_{2}(x, t), x \in[0,1], t \in(0,0.5],
\end{aligned}
$$


and the corresponding forcing terms $g_{1}(x, t)$ and $g_{2}(x, t)$ are of the form

$$
\begin{aligned}
& g_{1}(x, t)=e^{-i t}\left(i u_{1}(x, 0)-\varepsilon_{1}(-\Delta)^{\frac{\alpha}{2}} u_{1}(x, 0)+u_{2}(x, 0)+u_{1}(x, 0)+\left(\left|u_{1}(x, 0)\right|^{2}+\left|u_{2}(x, 0)\right|^{2}\right) u_{1}(x, 0)\right), \\
& g_{2}(x, t)=e^{-i t}\left(i u_{2}(x, 0)-\varepsilon_{2}(-\Delta)^{\frac{\alpha}{2}} u_{2}(x, 0)+u_{2}(x, 0)+u_{1}(x, 0)+\left(\left|u_{1}(x, 0)\right|^{2}+\left|u_{2}(x, 0)\right|^{2}\right) u_{2}(x, 0)\right),
\end{aligned}
$$

to obtain an exact solutions $u_{1}(x, t)=e^{-i t} x^{5}$ and $u_{2}(x, t)=e^{-i t} x^{5}$ with $\alpha=1.1, \varepsilon_{1}=\frac{\Gamma(6-\alpha)}{2 \Gamma(6)}, \varepsilon_{2}=\frac{\Gamma(6-\alpha)}{2 \Gamma(6)}$. The errors and order of convergence are listed in Tables 5 and 6 , confirming optimal $O\left(h^{N+1}\right)$ order of convergence across.

\begin{tabular}{|c||cc||c||cc||c||cc|}
\hline \hline $\mathrm{N}$ & \multicolumn{2}{|c}{$\mathrm{N}=1$} & \multicolumn{4}{c}{$\mathrm{N}=2$} & \multicolumn{2}{c|}{$\mathrm{N}=3$} \\
\hline \hline $\mathrm{K}$ & $L^{2}$-Error & order & $\mathrm{K}$ & $L^{2}$-Error & order & $\mathrm{K}$ & $L^{2}$-Error & order \\
\hline 10 & $3.95 \mathrm{e}-03$ & - & 10 & $4.7 \mathrm{e}-04$ & - & 10 & $1.02 \mathrm{e}-04$ & - \\
20 & $1.02 \mathrm{e}-03$ & 1.95 & 20 & $8.95 \mathrm{e}-05$ & 3.19 & 20 & $5.91 \mathrm{e}-06$ & 4.11 \\
40 & $2.21 \mathrm{e}-04$ & 2.20 & 40 & $1.05 \mathrm{e}-05$ & 3.09 & 40 & $3.82 \mathrm{e}-07$ & 3.95 \\
\hline \hline
\end{tabular}

Table 5: $L^{2}$-Error and order of convergence for $u_{1}$ with $K$ elements and polynomial order $N$.

\begin{tabular}{|c||cc||c||cc||c||cc|}
\hline \hline $\mathrm{N}$ & \multicolumn{2}{|c}{$\mathrm{N}=1$} & \multicolumn{4}{c}{$\mathrm{N}=2$} & \multicolumn{2}{c|}{$\mathrm{N}=3$} \\
\hline \hline $\mathrm{K}$ & $L^{2}$-Error & order & $\mathrm{K}$ & $L^{2}$-Error & order & $\mathrm{K}$ & $L^{2}$-Error & order \\
\hline 10 & $4.32 \mathrm{e}-03$ & - & 10 & $4.18 \mathrm{e}-04$ & - & 10 & $2.18 \mathrm{e}-04$ & - \\
20 & $1.17 \mathrm{e}-03$ & 1.89 & 20 & $9.04 \mathrm{e}-05$ & 3.24 & 20 & $1.32 \mathrm{e}-05$ & 4.05 \\
40 & $2.69 \mathrm{e}-04$ & 2.23 & 40 & $1.1 \mathrm{e}-05$ & 3.04 & 40 & $8.57 \mathrm{e}-07$ & 3.95 \\
\hline
\end{tabular}

Table 6: $L^{2}$-Error and order of convergence for $u_{2}$ with $K$ elements and polynomial order $N$.

Example 8.10. We consider the strongly coupled system as follows

$$
\begin{aligned}
& i \frac{\partial u_{1}}{\partial t}-(-\Delta)^{\frac{\alpha}{2}} u_{1}+\left(\left|u_{1}\right|^{2}+\left|u_{2}\right|^{2}\right) u_{1}+u_{1}+\varpi_{1} u_{2}=0 \\
& i \frac{\partial u_{2}}{\partial t}-(-\Delta)^{\frac{\alpha}{2}} u_{2}+\left(\left|u_{1}\right|^{2}+\left|u_{2}\right|^{2}\right) u_{2}+\varpi_{1} u_{1}+u_{2}=0
\end{aligned}
$$

subject to the initial conditions

$$
\begin{aligned}
& u_{1}(x, 0)=\sqrt{2} r_{1} \operatorname{sech}\left(r_{1} x+D\right) e^{i V_{0} x}, \\
& u_{2}(x, 0)=\sqrt{2} r_{2} \operatorname{sech}\left(r_{2} x+D\right) e^{i V_{0} x},
\end{aligned}
$$


where $r_{1}=r_{2}=1, V_{0}=0.4, D=10$ and $x \in[-40,40]$.

Figures 6 and 7 shows that the proposed scheme simulates the solitary waves well. The two waves emerge without any changes in their shapes for any $1<\alpha \leq 2$. This phenomenon shows that the interaction is elastic. The Figures 810 present the numerical solutions for different values of order $\alpha$ for fixed $\varpi_{1}=0.0175$. From these figures it is obvious that the collision is always inelastic. That is, the shapes and directions of two waves have changed after interaction.

Example 8.11. Finally, we consider the following weakly coupled problem

$$
\begin{aligned}
& i \frac{\partial u_{1}}{\partial t}-(-\Delta)^{\frac{\alpha}{2}} u_{1}+\left(\left|u_{1}\right|^{2}+\beta\left|u_{2}\right|^{2}\right) u_{1}=0 \\
& i \frac{\partial u_{2}}{\partial t}-(-\Delta)^{\frac{\alpha}{2}} u_{2}+\left(\beta\left|u_{1}\right|^{2}+\left|u_{2}\right|^{2}\right) u_{2}=0
\end{aligned}
$$

subject to the initial conditions

$$
\begin{aligned}
& u_{1}(x, 0)=\sqrt{2} r_{1} \operatorname{sech}\left(r_{1} x+D\right) e^{i V_{0} x}, \\
& u_{2}(x, 0)=\sqrt{2} r_{2} \operatorname{sech}\left(r_{2} x+D\right) e^{i V_{0} x},
\end{aligned}
$$

when $\beta=1$ and $\alpha=2$, the problem collapses to the Manakov equation, and the solitary waves collide elastically see Figure 11. The exact solutions are given by

$$
\begin{gathered}
u_{1}(x, t)=\sqrt{2} r_{1} \operatorname{sech}\left(r_{1} x-2 r_{1} V_{0} t+D\right) e^{i\left(V_{0} x+\left(r_{1}^{2}-V_{0}^{2}\right) t\right)}, \\
u_{2}(x, t)=\sqrt{2} r_{2} \operatorname{sech}\left(r_{2} x-2 r_{2} V_{0} t-D\right) e^{i\left(-V_{0} x+\left(r_{2}^{2}-V_{0}^{2}\right) t\right)},
\end{gathered}
$$

where $r_{1}=1, r_{2}=1, V_{0}=0.4, D=10$ and $x \in[-40,40]$. The Figures 12 and 13 present the numerical solutions for different values of order $\alpha$ and $\beta$. From these figures it is obvious that the collision of solitons are inelastic. In particular, the colliding particles stick together after interaction when $\alpha=1.8$, which means that there may occur a completely inelastic collision see Figure 13. 
$|u$,

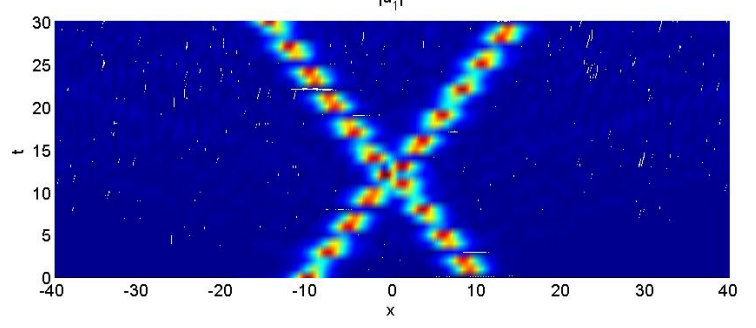

$\left|u_{2}\right|$

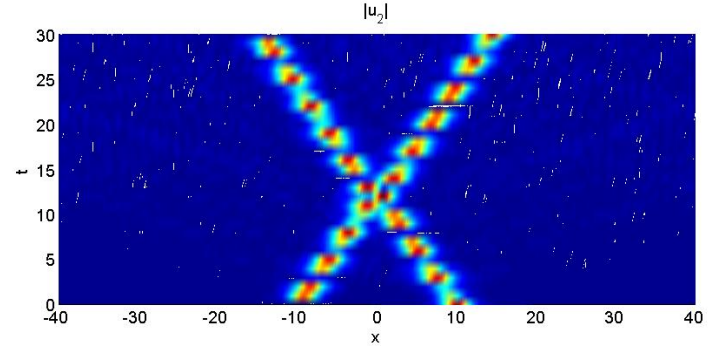

$\left|u_{1}\right|+\left|u_{2}\right|$

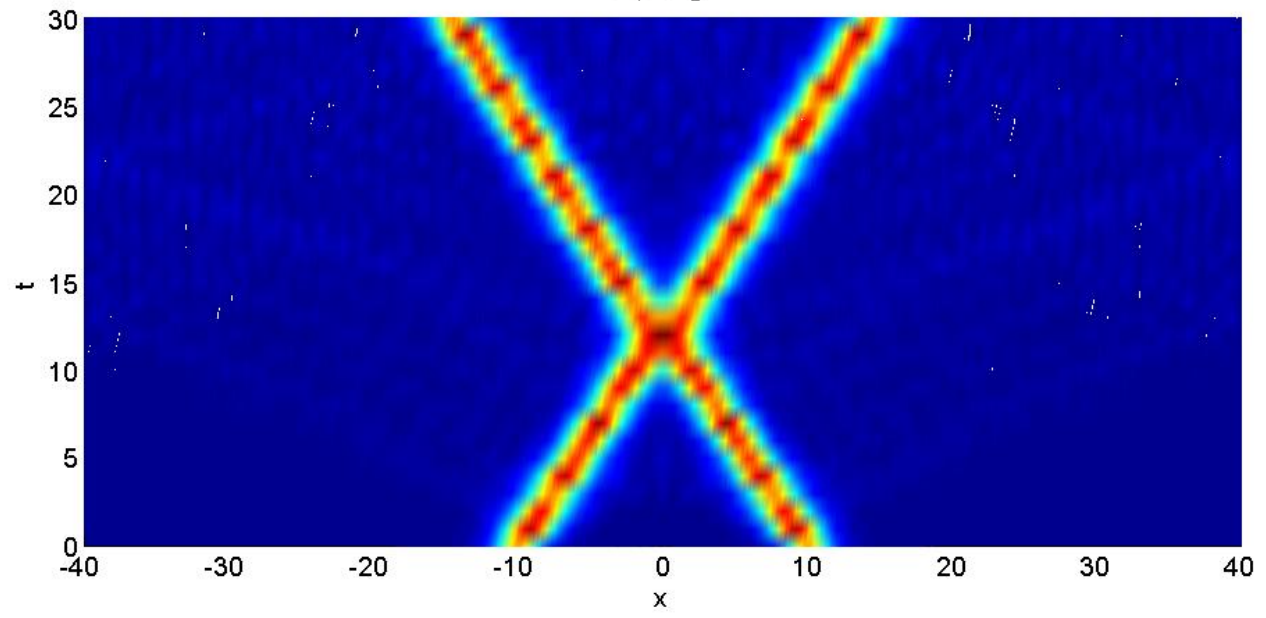

Figure 6: The numerical simulation of the two soliton waves for Example 8.10 with $\varpi_{1}=1, \alpha=2$. 

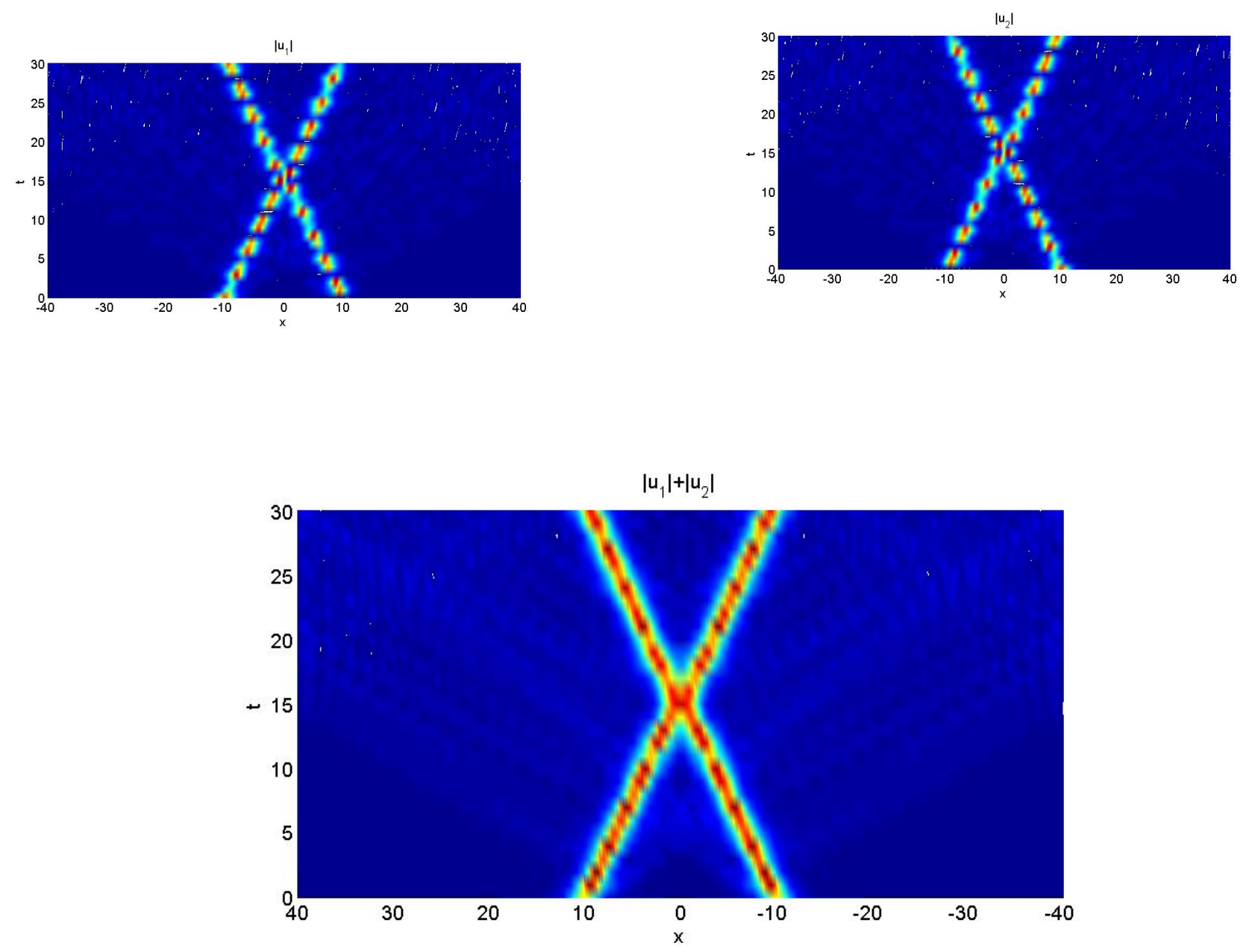

Figure 7: The numerical simulation of the two soliton waves for Example 8.10 with $\varpi_{1}=1, \alpha=1.6$. 

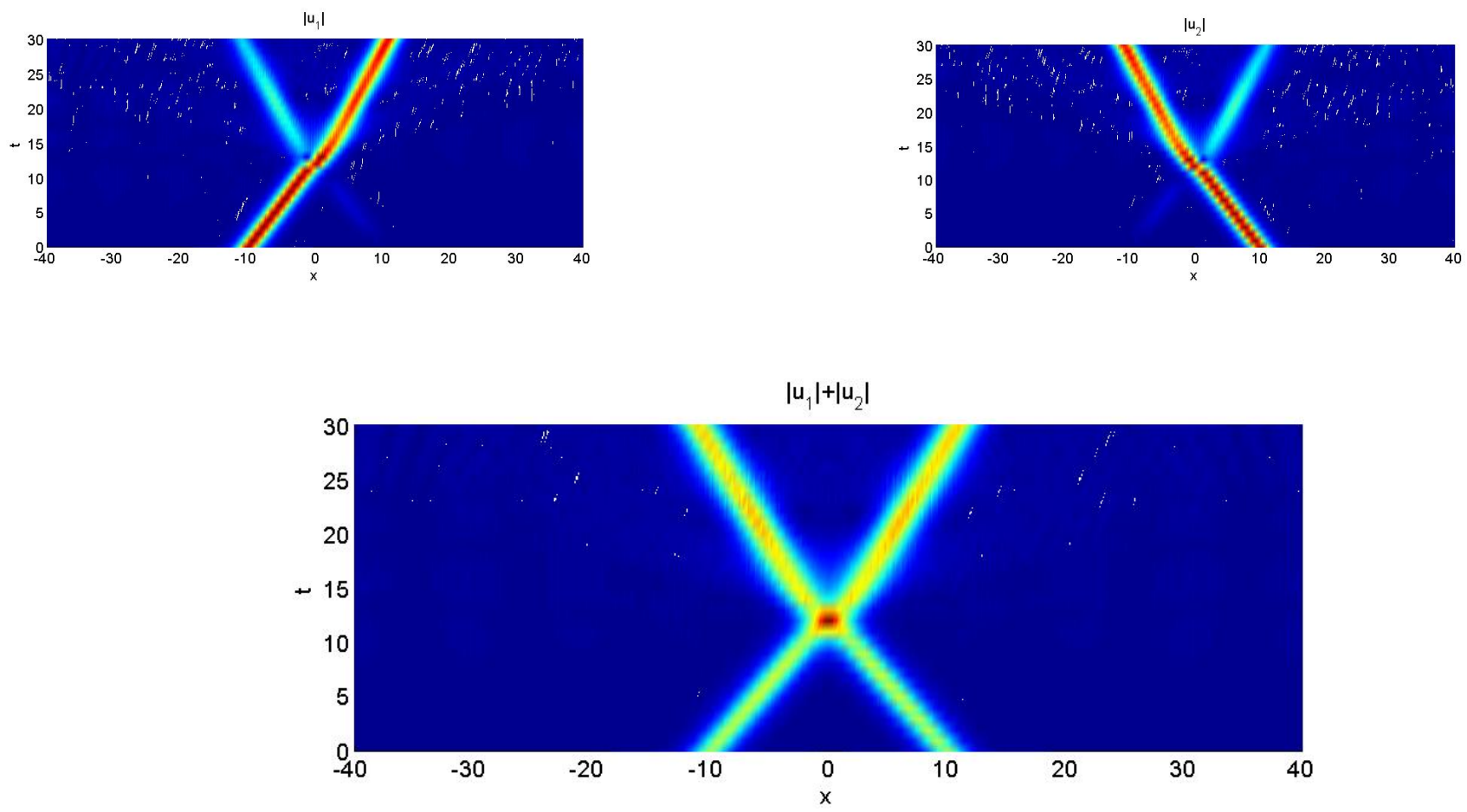

Figure 8: The numerical simulation of the two soliton waves for Example 8.10 with $\varpi_{1}=0.0175$ and $\alpha=2$. 

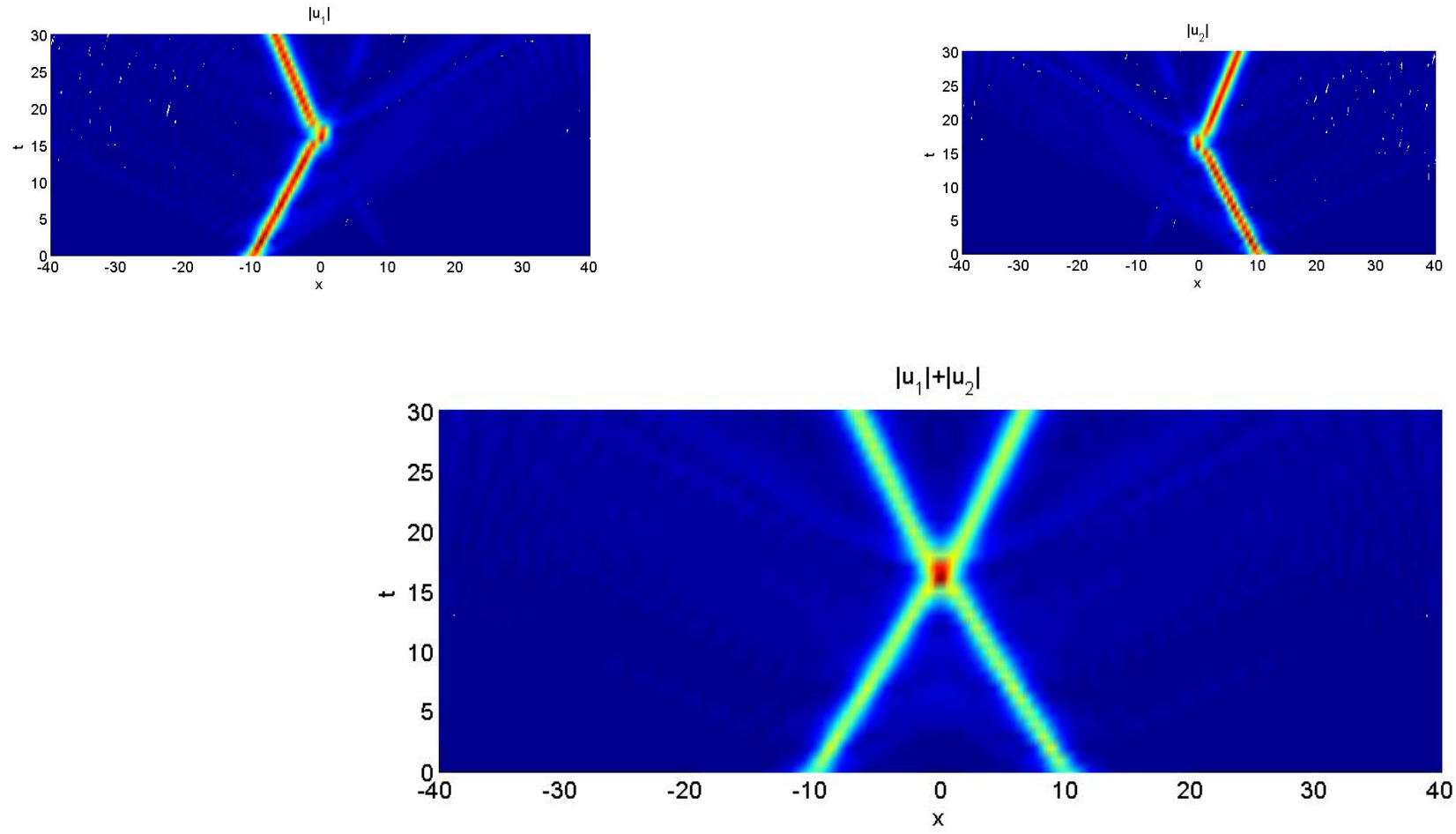

Figure 9: The numerical simulation of the two soliton waves for Example 8.10 with $\varpi_{1}=0.0175$ and $\alpha=1.6$. 

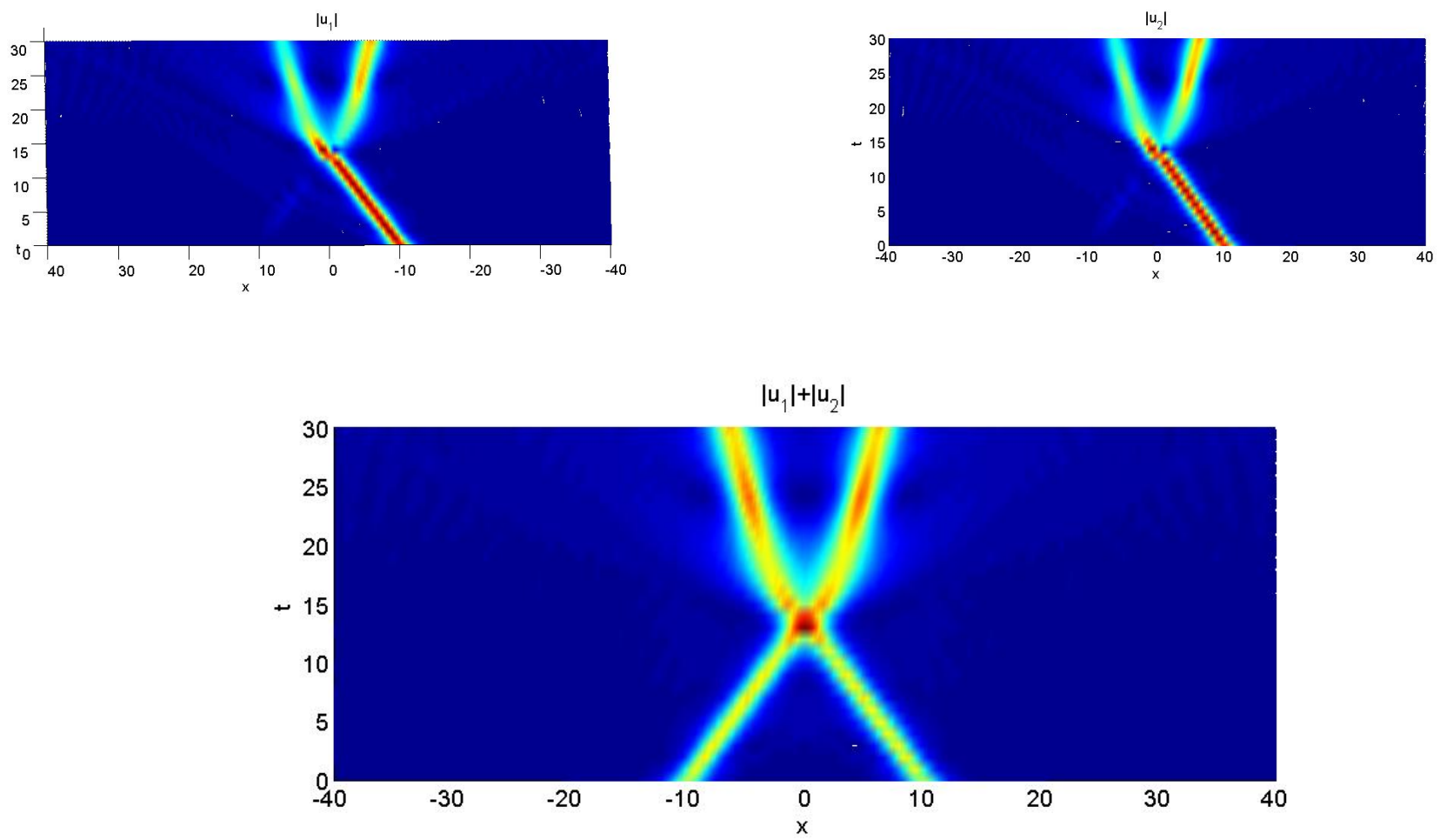

Figure 10: The numerical simulation of the two soliton waves for Example 8.10 with $\varpi_{1}=0.0175$ and $\alpha=1.8$. 

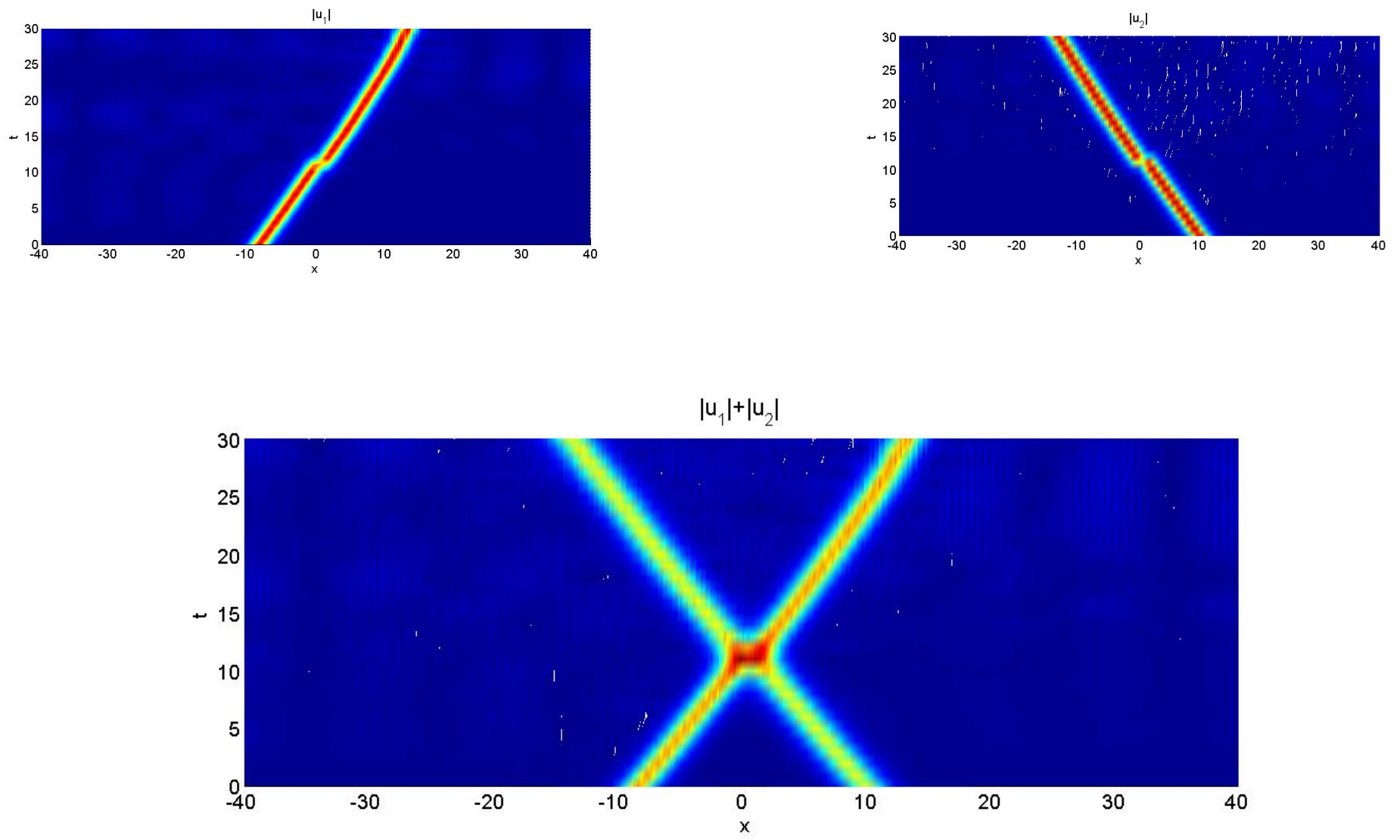

Figure 11: The numerical simulation of the two soliton waves for Example 8.11 with $\beta=1$ and $\alpha=2$. 

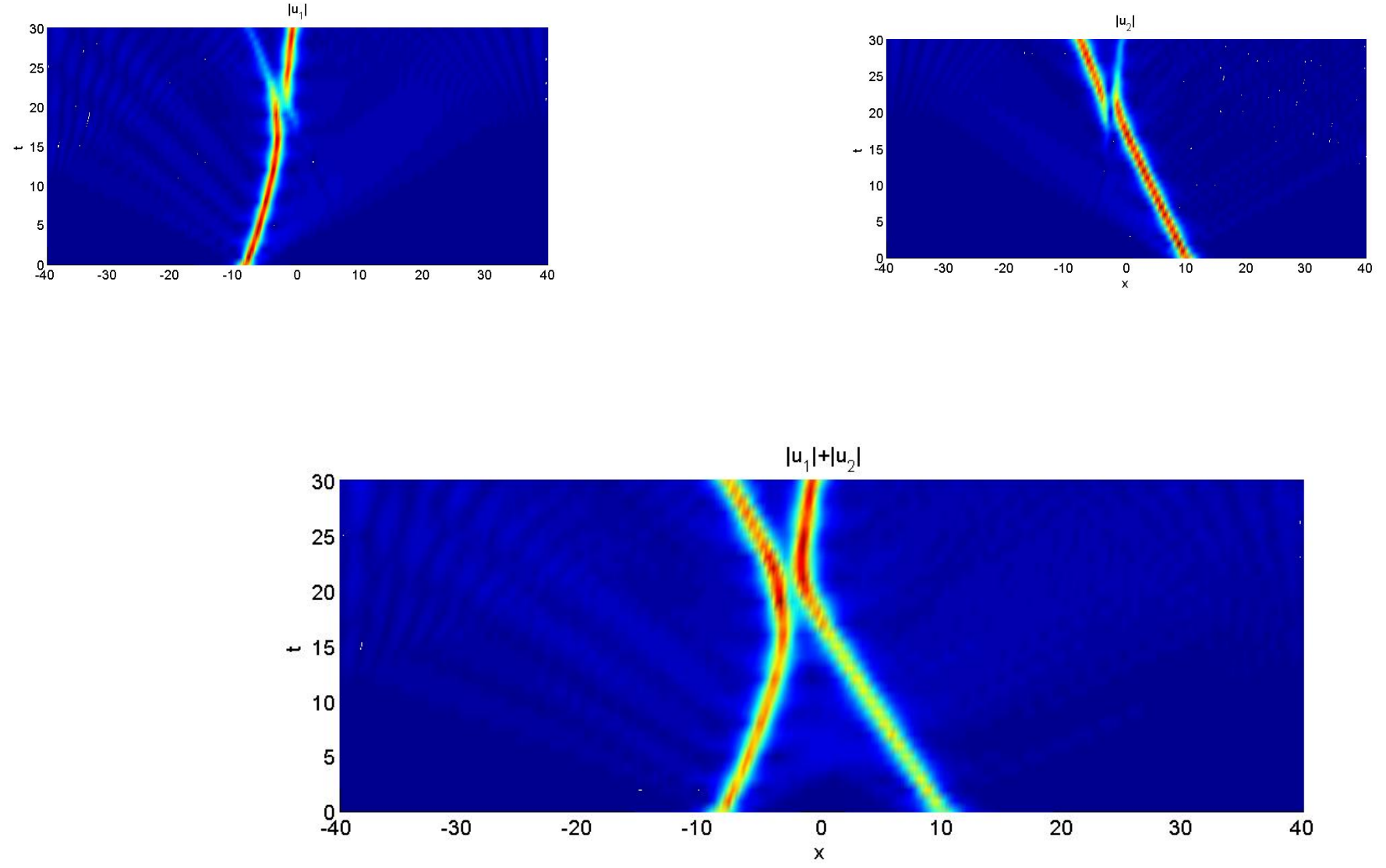

Figure 12: The numerical simulation of the two soliton waves for Example 8.11 with $\beta=1$ and $\alpha=1.6$. 

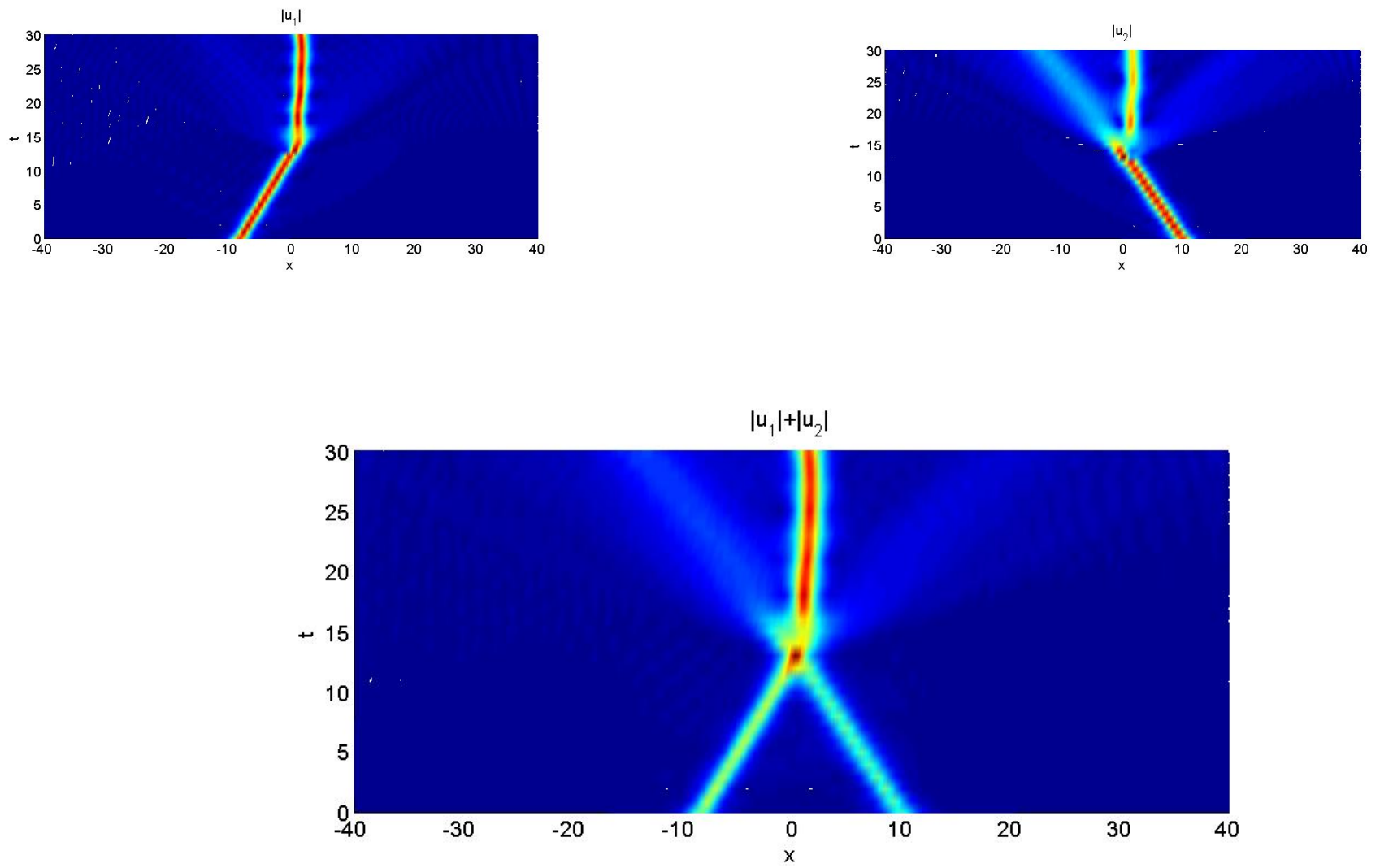

Figure 13: The numerical simulation of the two soliton waves for Example 8.11 with $\beta=0.3$ and $\alpha=1.8$. 


\section{Conclusions}

We propose a DDG finite element method for solving fractional convection-diffusion and Schrödinger type equations. The scheme is formulated using the direct weak for these problems and the construct of the suitable numerical flux on the cell edges. Unlike the traditional LDG method, the method in this paper is applied without introducing any auxiliary variables or rewriting the original equation into a 1st order system. An DDG method is proposed and stability and a priori $L^{2}$ error estimates are presented. Numerical experiments for the fractional convection-diffusion and Schrödinger type equations confirm the analysis. The numerical tests demonstrate both accuracy and capacity of these methods, in particular, the numerical results are accurate for long time simulation.

\section{References}

\section{References}

[1] B. B. Mandelbrot, R. Pignoni, The fractal geometry of nature, WH freeman New York, 1983.

[2] V. E. Tarasov, Fractional dynamics: applications of fractional calculus to dynamics of particles, fields and media, Springer Science \& Business Media, 2011.

[3] R. Herrmann, Fractional calculus: an introduction for physicists, World Scientific, 2014.

[4] V. V. Uchaikin, Fractional derivatives for physicists and engineers, Springer, 2013.

[5] H. K. Moffatt, G. Zaslavsky, P. Comte, M. Tabor, Topological aspects of the dynamics of fluids and plasmas, volume 218, Springer Science \& Business Media, 2013.

[6] G. M. Zaslavsky, Chaos, fractional kinetics, and anomalous transport, Physics Reports 371 (2002) 461-580.

[7] A. I. Saichev, G. M. Zaslavsky, Fractional kinetic equations: solutions and applications, Chaos: An Interdisciplinary Journal of Nonlinear Science 7 (1997) 753-764.

[8] G. Zaslavsky, M. Edelman, Weak mixing and anomalous kinetics along filamented surfaces, Chaos: An Interdisciplinary Journal of Nonlinear Science 11 (2001) 295-305.

[9] R. Metzler, J. Klafter, The restaurant at the end of the random walk: recent developments in the description of anomalous transport by fractional dynamics, Journal of Physics A: Mathematical and General 37 (2004) R161.

[10] K. B. Oldham, Fractional differential equations in electrochemistry, Advances in Engineering Software 41 (2010) 9-12.

[11] E. Cuesta, M. Kirane, S. A. Malik, Image structure preserving denoising using generalized fractional time integrals, Signal Processing 92 (2012) 553-563. 
[12] J. Cai, Multisymplectic schemes for strongly coupled Schrödinger system, Applied Mathematics and Computation 216 (2010) 2417-2429.

[13] A. El-Sayed, M. Gaber, On the finite caputo and finite Riesz derivatives, Electronic Journal of Theoretical Physics 3 (2006) 81-95.

[14] S. I. Muslih, O. P. Agrawal, Riesz fractional derivatives and fractional dimensional space, International Journal of Theoretical Physics 49 (2010) 270-275.

[15] Q. Yang, F. Liu, I. Turner, Numerical methods for fractional partial differential equations with Riesz space fractional derivatives, Applied Mathematical Modelling 34 (2010) 200-218.

[16] A. C. Fowler, Evolution equations for dunes and drumlins, Rev. R. Acad. de Cien, Serie A. Mat 96 (2002) $377-387$.

[17] M. Alfaro, J. Droniou, General fractal conservation laws arising from a model of detonations in gases, Applied Mathematics Research eXpress 2012 (2012) 127-151.

[18] P. Clavin, Instabilities and nonlinear patterns of overdriven detonations in gases, in: Nonlinear PDEs in Condensed Matter and Reactive Flows, Springer, 2002, pp. 49-97.

[19] P. Azerad, A. Bouharguane, J.-F. Crouzet, Simultaneous denoising and enhancement of signals by a fractal conservation law, Communications in Nonlinear Science and Numerical Simulation 17 (2012) 867-881.

[20] M. F. Shlesinger, G. M. Zaslavsky, U. Frisch, Lévy flights and related topics in physics, in: Levy flights and related topics in Physics, volume 450, 1995.

[21] M. Cui, A high-order compact exponential scheme for the fractional convection-diffusion equation, Journal of Computational and Applied Mathematics 255 (2014) 404-416.

[22] Y. Lin, C. Xu, Finite difference/spectral approximations for the time-fractional diffusion equation, Journal of Computational Physics 225 (2007) 1533-1552.

[23] Z. Wang, S. Vong, A high-order exponential ADI scheme for two dimensional time fractional convectiondiffusion equations, Computers \& Mathematics with Applications 68 (2014) 185-196.

[24] S. Zhai, X. Feng, Y. He, An unconditionally stable compact ADI method for three-dimensional timefractional convection-diffusion equation, Journal of Computational Physics 269 (2014) 138-155.

[25] F. Liu, P. Zhuang, V. Anh, I. Turner, K. Burrage, Stability and convergence of the difference methods for the space-time fractional advection-diffusion equation, Applied Mathematics and Computation 191 (2007) $12-20$. 
[26] M. Chen, W. Deng, A second-order numerical method for two-dimensional two-sided space fractional convection diffusion equation, Applied Mathematical Modelling 38 (2014) 3244-3259.

[27] W. Deng, Finite element method for the space and time fractional Fokker-Planck equation, SIAM Journal on Numerical Analysis 47 (2008) 204-226.

[28] H.-f. Ding, Y.-x. Zhang, New numerical methods for the Riesz space fractional partial differential equations, Computers \& Mathematics with Applications 63 (2012) 1135-1146.

[29] A.-M. Matache, C. Schwab, T. P. Wihler, Fast numerical solution of parabolic integrodifferential equations with applications in finance, SIAM Journal on Scientific Computing 27 (2005) 369-393.

[30] R. Cont, E. Voltchkova, A finite difference scheme for option pricing in jump diffusion and exponential Lévy models, SIAM Journal on Numerical Analysis 43 (2005) 1596-1626.

[31] D. Benney, A. Newell, The propagation of nonlinear wave envelopes, Journal of mathematics and Physics 46 (1967) 133-139.

[32] R. Bullough, P. Jack, P. Kitchenside, R. Saunders, Solitons in laser physics, Physica Scripta 20 (1979) 364.

[33] J. Yang, Classification of the solitary waves in coupled nonlinear Schrödinger equations, Physica D: Nonlinear Phenomena 108 (1997) 92-112.

[34] M. Ran, C. Zhang, A conservative difference scheme for solving the strongly coupled nonlinear fractional Schrödinger equations, Communications in Nonlinear Science and Numerical Simulation 41 (2016) 64-83.

[35] P. Wang, C. Huang, An energy conservative difference scheme for the nonlinear fractional schrödinger equations, Journal of Computational Physics 293 (2015) 238-251.

[36] Z. Yang, A class of linearized energy-conserved finite difference schemes for nonlinear space-fractional Schrödinger equations, International Journal of Computer Mathematics 93 (2016) 609-626.

[37] M. Li, C. Huang, P. Wang, Galerkin finite element method for nonlinear fractional Schrödinger equations, Numerical Algorithms (2016) 1-27.

[38] L. Wei, X. Zhang, S. Kumar, A. Yildirim, A numerical study based on an implicit fully discrete local discontinuous Galerkin method for the time-fractional coupled Schrödinger system, Computers \& Mathematics with Applications 64 (2012) 2603-2615.

[39] F. Bassi, S. Rebay, A high-order accurate discontinuous finite element method for the numerical solution of the compressible Navier-Stokes equations, Journal of computational physics 131 (1997) 267-279. 
[40] B. Cockburn, C.-W. Shu, TVB Runge-Kutta local projection discontinuous Galerkin finite element method for conservation laws. II. General framework, Mathematics of computation 52 (1989) 411-435.

[41] B. Cockburn, C. Dawson, Approximation of the velocity by coupling discontinuous Galerkin and mixed finite element methods for flow problems, Computational Geosciences 6 (2002) 505-522.

[42] B. Cockburn, G. Kanschat, D. Schötzau, A locally conservative LDG method for the incompressible NavierStokes equations, Mathematics of Computation 74 (2005) 1067-1095.

[43] K. Mustapha, W. McLean, Piecewise-linear, discontinuous Galerkin method for a fractional diffusion equation, Numerical Algorithms 56 (2011) 159-184.

[44] K. Mustapha, W. McLean, Uniform convergence for a discontinuous Galerkin, time-stepping method applied to a fractional diffusion equation, IMA Journal of Numerical Analysis 32 (2012) 906-925.

[45] K. Mustapha, W. McLean, Superconvergence of a discontinuous Galerkin method for fractional diffusion and wave equations, SIAM Journal on Numerical Analysis 51 (2013) 491-515.

[46] W. Deng, J. S. Hesthaven, Local discontinuous Galerkin methods for fractional diffusion equations, ESAIM: Mathematical Modelling and Numerical Analysis 47 (2013) 1845-1864.

[47] Q. Xu, J. S. Hesthaven, Discontinuous Galerkin method for fractional convection-diffusion equations, SIAM Journal on Numerical Analysis 52 (2014) 405-423.

[48] T. Aboelenen, H. El-Hawary, A high-order nodal discontinuous Galerkin method for a linearized fractional Cahn-Hilliard equation, Computers \& Mathematics with Applications 73 (2017) 1197-1217.

[49] T. Aboelenen, A high-order nodal discontinuous Galerkin method for nonlinear fractional Schrödinger type equations, Communications in Nonlinear Science and Numerical Simulation 54 (2018) 428 - 452.

[50] C. Huang, X. Yu, C. Wang, Z. Li, N. An, A numerical method based on fully discrete direct discontinuous Galerkin method for the time fractional diffusion equation, Applied Mathematics and Computation 264 (2015) 483-492.

[51] H. Liu, J. Yan, The direct discontinuous Galerkin (DDG) methods for diffusion problems, SIAM Journal on Numerical Analysis 47 (2009) 675-698.

[52] H. Liu, J. Yan, The direct discontinuous Galerkin (DDG) method for diffusion with interface corrections, Communications in Computational Physics 8 (2010) 541.

[53] H. Liu, Optimal error estimates of the direct discontinuous Galerkin method for convection-diffusion equations, Mathematics of Computation 84 (2015) 2263-2295. 
[54] K. Miller, B. Ross, An Introduction to the Fractional Calculus and Fractional Differential Equations, Wiley, 1993. URL: https://books.google.co.in/books?id=MOp_QgAACAAJ.

[55] V. J. Ervin, J. P. Roop, Variational formulation for the stationary fractional advection dispersion equation, Numerical Methods for Partial Differential Equations 22 (2006) 558-576.

[56] A. A. Kilbas, H. M. Srivastava, J. J. Trujillo, Theory and Applications of Fractional Differential Equations, Volume 204 (North-Holland Mathematics Studies), Elsevier Science Inc., New York, NY, USA, 2006.

[57] P. G. Ciarlet, Finite Element Method for Elliptic Problems, Society for Industrial and Applied Mathematics, Philadelphia, PA, USA, 2002.

[58] Q. Zhang, C.-W. Shu, Error estimates to smooth solutions of Runge-Kutta discontinuous Galerkin methods for scalar conservation laws, SIAM Journal on Numerical Analysis 42 (2004) 641-666.

[59] B. Cockburn, High-Order Methods for Computational Physics, Springer Berlin Heidelberg, Berlin, Heidelberg, 1999, pp. 69-224. URL: http://dx.doi.org/10.1007/978-3-662-03882-6_2. doi 10.1007/ 978-3-662-03882-6_2.

[60] S. Gottlieb, C.-W. Shu, Total variation diminishing Runge-Kutta schemes, Math. Comput. 67 (1998) 73-85. 